\title{
THE STRUCTURE OF JET TURBULENCE PRODUCING JET NOISE
}

Prepared for:

NATIONAL AERONAUTICS AND SPACE ADMINISTRATION HEADQUARTERS CONTRACT DIVISION WASHINGTON, D.C.

Attention: CHIEF, FLUID DYNAMICS BRANCH, CODE RAA OFFICE OF ADVANCED RESEARCH AND TECHNOLOGY

CONTRACT NASW-1938

(NASA-CR-126483) THE STRUCTORE OF JET TURBULENCE PRODUCING JET NOISE Annual Report C.E. Wooldridge, et al (Stanford Research Inst.) Jun. 197172 p CSCL 20A 玄 (NASA CR OR TMX OR AD NUMBER)
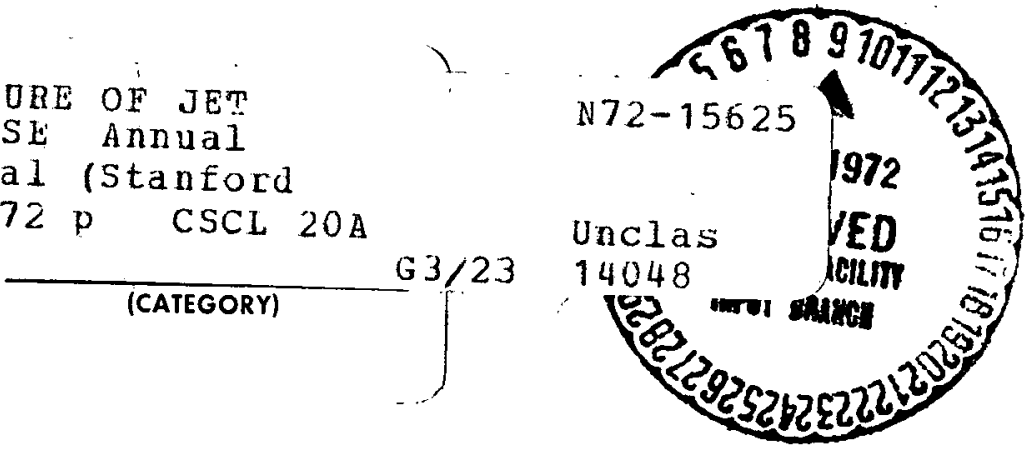


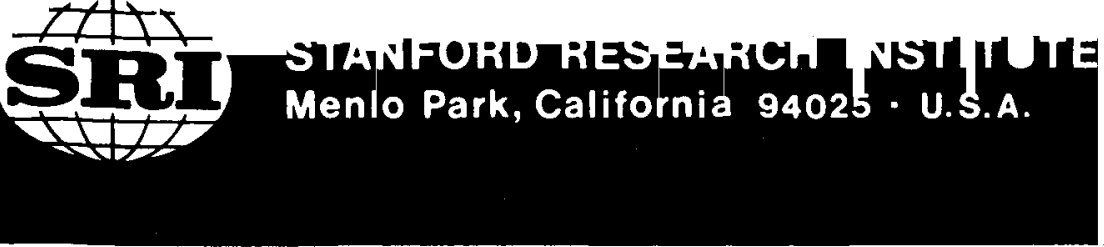

Annual Report

June 1971

\section{THE STRUCTURE OF JET TURBULENCE PRODUCING JET NOISE}

By: C. E. WOOLDRIDGE, D. C. WOOTEN, and A. J. AMARO

Prepared for:

NATIONAL AERONAUTICS AND SPACE ADMINISTRATION HEADQUARTERS CONTRACT DIVISION WASHINGTON, D.C.

Attention: CHIEF, FLUID DYNAMICS BRANCH, CODE RAA OFFICE OF ADVANCED RESEARCH AND TECHNOLOGY

CONTRACT NASW-1938

SRI Project SCU 8139

Approved by:

E. C. WOOD

Executive Director SRI-Irvine 


\title{
PRECEOING PAGE BLANK NOT FILMED
}

\begin{abstract}
A BSTRACT
The structure of the turbulence in the mixing region for the first few diameters downstream from the outlet of a circular subsonic jet is characterized at three Mach numbers, $0.3,0.5$, and 0.7 , with most of the measurements taken at $M=0.3$. Profiles of turbulence intensity showed that downstream of the lip intensity is independent of axial distance, while in the core intensity varies by a factor of eight between the jet outlet and the end of the core. A digital data reduction program was used to calculate the auto- and cross-correlations of axial velocity fluctuations and the power spectral densities. Convection velocities were measured using broad band, hot wire signals and signals that were digitally filtered for band-passes about center frequencies of $0.8,1.3,1.6$, and $3.2 \mathrm{kHz}$. The center frequency of $1.3 \mathrm{kHz}$ corresponded to the peak energy in the core spectrum. The convection velocities of the lower frequency components of the signals were lower and varied more slowly with radial position than the convection velocities of the higher frequency components and the overall signal. Thus, the large eddies corresponding to the low frequency components tended to be convected at more nearly a constant velocity across the shear layer. The results support the hypothesis that the coherent pressure field is driven by the intermittent fluctuations at the core boundary, which in turn are related to the large (low frequency) eddies.
\end{abstract}


PREFACE

This second-year study of the structure of turbulence in the noiseproducing region of a round subsonic jet was performed principally by the authors. Thanks are due to Dr. R. C. Singleton for writing the computer program used to carry out the digital analysis, to Mr. Bruce Craig for helping with the analog-to-digital conversion of the hot wire data, and to $\mathrm{Mr}$. Gary Plapp for spending many long hours in the laboratory.

This project was under the initial management of Mr. Ira R. Schwartz, Chief, Fluid Mechanics Branch, NASA Headquarters, Washington, D.C.; the management was taken over by $\mathrm{Dr}$. William H. Roudebush in March 1971 . 
CONTENTS

\begin{abstract}
. . . . . . . . . . . . . . . . . . . . . . . $i i i$
PREFACE . . . . . . . . . . . . . . . . . . . . . . . $\mathrm{v}$

LIST OF ILLUSTRATIONS . . . . . . . . . . . . . . . . . . . ix

NOMENCLATURE. . . . . . . . . . . . . . . . . . . . . . . . . . xi

I INTRODUCTION. . . . . . . . . . . . . . . . . . . 1

II MEAN PROFILE MEASUREMENTS . . . . . . . . . . . . . . . 5

II POWER SPECTRAL DENSITY MEASUREMENTS . . . . . . . . . . . . 11

IV CROSS-CORRELATION MEASUREMENTS. . . . . . . . . . . . . . 23

V CONVECTION VELOCITY MEASUREMENTS. . . . . . . . . . . . . . . 39

VI THE EFFECT OF EVAPORATING DROPLETS ON SOUND 51

GENERATION BY JET TURBULENCE。 . . . . . . . . . . . . . .

VII CONCLUDING REMARKS. . . . . . . . . . . . . . . . . . 59

REFERENCES. . . . . . . . . . . . . . . . . . . . 61
\end{abstract}




\title{
PRECEDING PAGE BLANK NOT FILMED
}

\author{
LIST OF ILLUSTRATIONS
}

Figure 1 Schematic Diagram of Jet Noise Test System . . . . . . 3

Figure 2 Mean Velocity Profiles at Various Mach Numbers at $\mathrm{X} / \mathrm{D}=2 .$. . . . . . . . . . . . . . . .

Figure 3 Variation of Mean Velocity with Axial Distance, $\mathrm{M}=0.3$.

Figure 4 Turbulence Intensity and Mean Velocity Profiles at Jet Exit, $M=0.3, X / D=0.042 . .$. . . . . . .

Figure 5 Variation of Turbulence Intensity with Axial Distance,

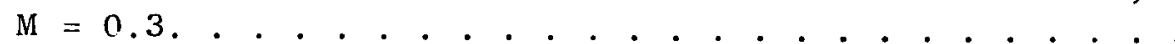

Figure 6 Power Spectral Density at Different Radial Positions, $\mathrm{M}=0.3$. . . . . . . . . . . . . . . . . 12

Figure 7 Variation of Energy in Band Centered at $1300 \mathrm{~Hz}$ with Axial Distance Along Jet Axis, $M=0.3$, Filter Bandwidth $=10 \mathrm{~Hz}$. . . . . . . . . . . . . . . . . .

Figure 8 Power Spectral Density at Different Mach Numbers,

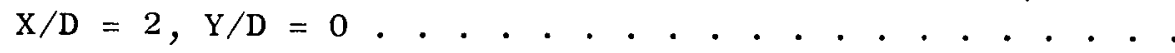

Figure 9 Velocity Profile in the Settling Chamber, $M=0.7 . .$. .

Figure 10 Power Spectral Densities with Modified Nozzles, $\mathrm{X} / \mathrm{D}=2, \mathrm{M}=0.3 . . . . . . . . . . . . .$.

Figure 11 Computer Printout of Hot Wire Signal,

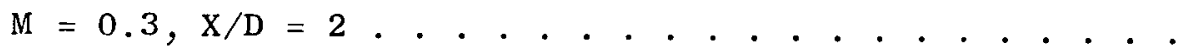

Figure 12 Computer Printout of Power Spectral Density, $\mathrm{M}=0.3, \mathrm{X} / \mathrm{D}=2, \mathrm{Y} / \mathrm{D}=0.133 . . . . . . . . . .$. .

Figure 13 Computer Printout of Typical Cross-Correlation, $\mathrm{M}=0.3, \mathrm{X} / \mathrm{D}=2, \mathrm{Y} / \mathrm{D}=0.4 . . . . . . . . .$. . 


\section{LIST OF ILLUSTRATIONS (concluded)}

Figure 14 Cross-Correlation of Hot Wire Signals in Jet Core Versus Time Delay for Selected Hot Wire Spacings, $\mathrm{M}=0.3, \mathrm{Y} / \mathrm{D}=0.133$, Upstream Wire at $\mathrm{X} / \mathrm{D}=2 . . . .30$

Figure 15 Cross-Correlation of Hot Wire Signals in Jet Core Versus Time Delay for Selected Hot Wire Spacings, $\mathrm{M}=0.3, \mathrm{Y} / \mathrm{D}=0$, Upstream Wire at $\mathrm{X} / \mathrm{D}=4 . . . .$.

Figure 16 Cross-Correlations of Axial Velocity Fluctuation Versus Time Delay for Selected Hot Wire Spacings, $\mathrm{M}=0.3, \mathrm{Y} / \mathrm{D}=0.4$, Upstream Wire at $\mathrm{X} / \mathrm{D}=2 . . .$.

Figure 17 Cross-Correlations of Axial Velocity Fluctuation Versus Time Delay for Selected Hot Wire Spacings, $\mathrm{M}=0.3, \mathrm{Y} / \mathrm{D}=0.53$, Upstream Wire at $\mathrm{X} / \mathrm{D}=4 . . .$.

Figure 18 Convection Velocity Versus Wire Spacing. . . . . . .

Figure 19 Convection Velocity Profiles Across the Jet,

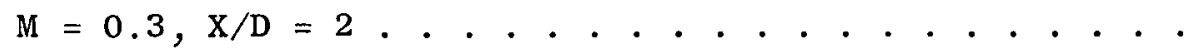

Figure 20 Convection Velocity Profiles Across the Jet,

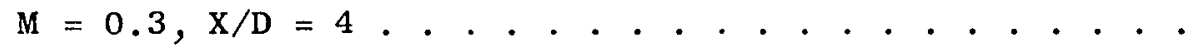

Figure 21 Convection Velocity Profiles Across the Jet, Asymptotic Values for Large Wire Spacing, $M=0.5$,

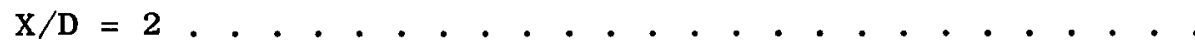

Figure 22 Space-Time Correlation Contours, $M=0.3, X / D=2$,

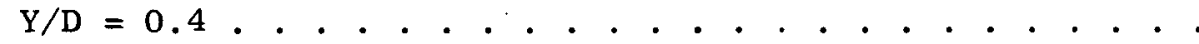




\section{NOMENCLATURE}

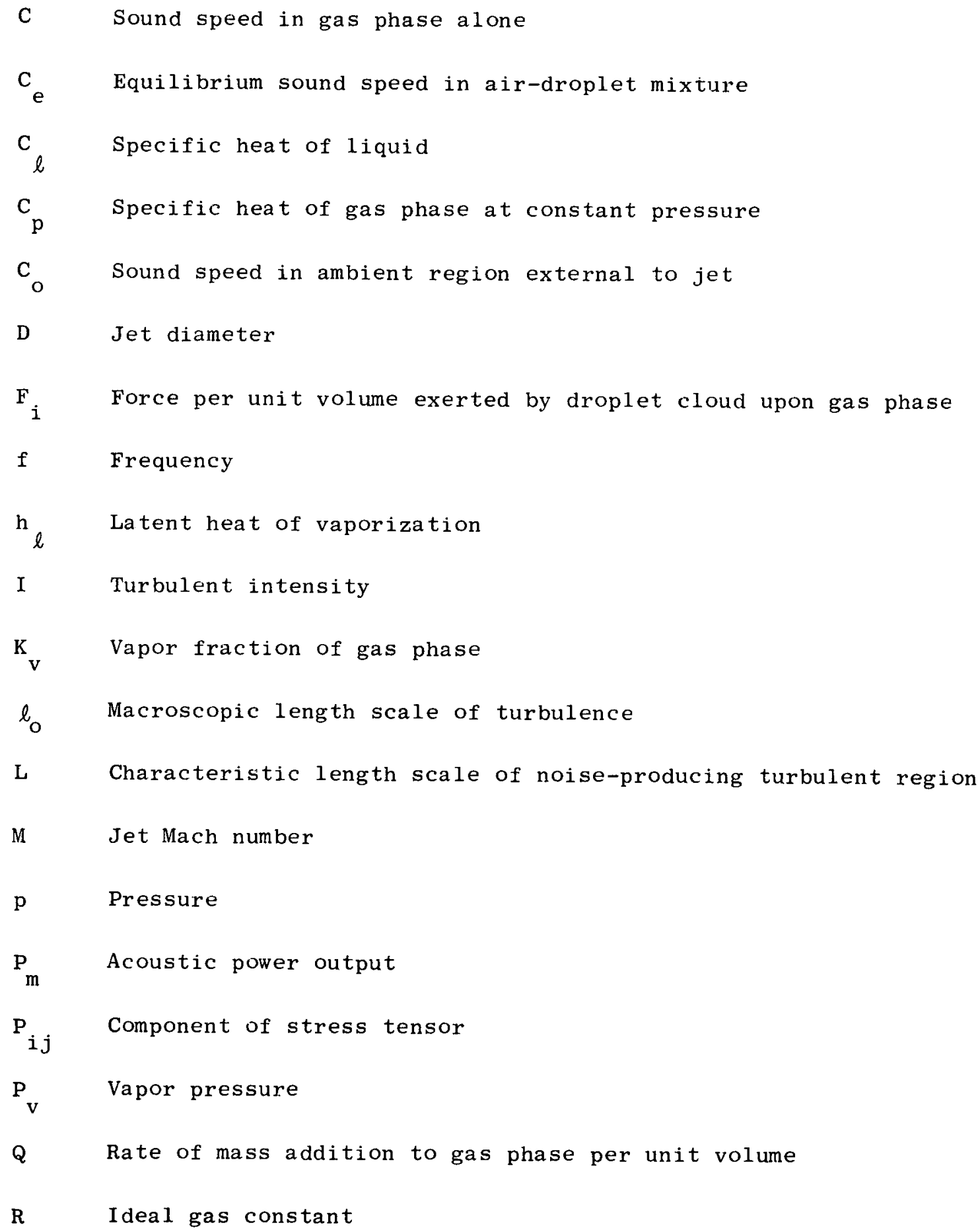




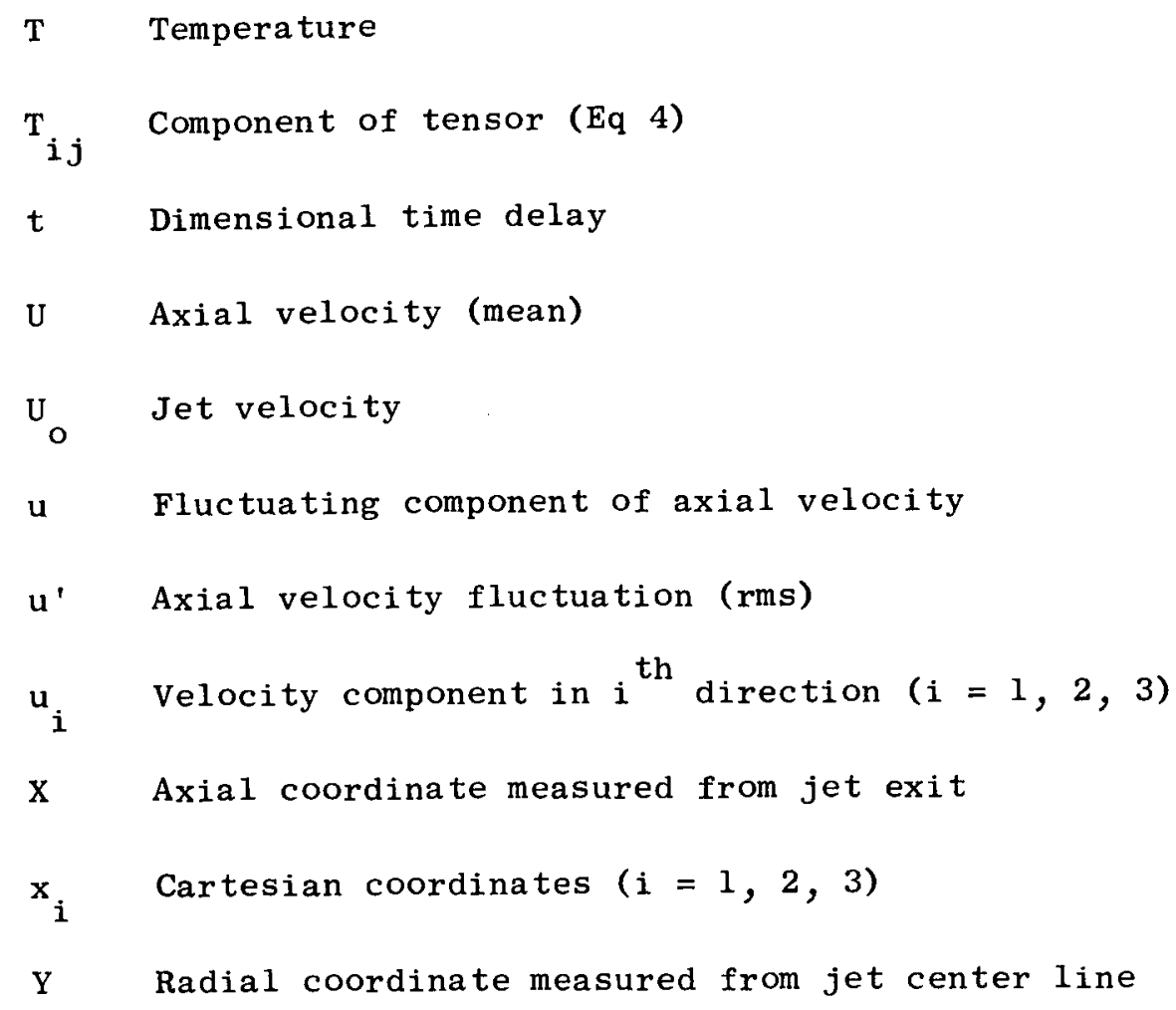

\section{Greek Symbols}

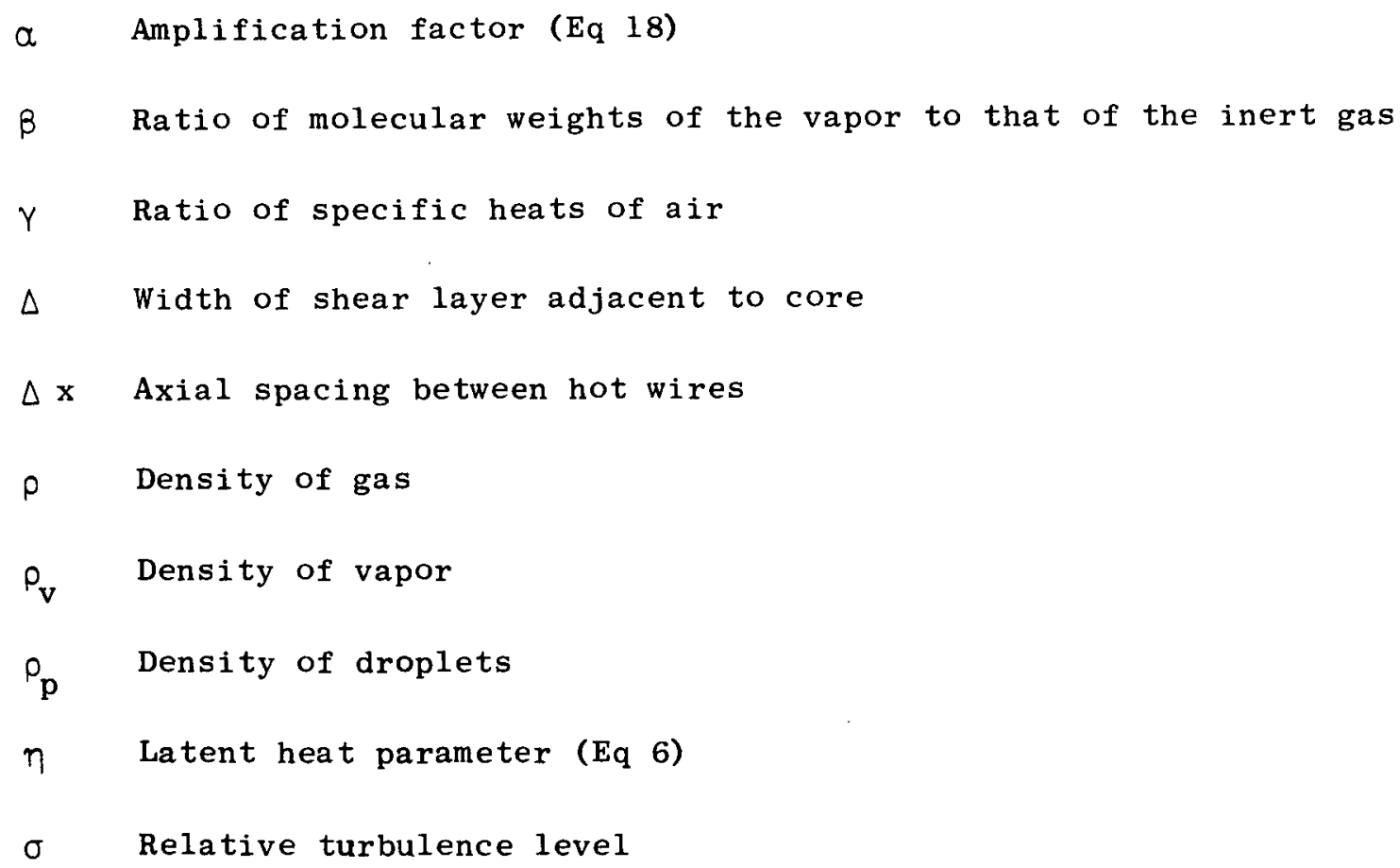




\section{INTRODUCTION}

Over the past two decades, the problem of aircraft noise has been studied both theoretically and experimentally. The theoretical groundwork was done primarily by Lighthill ${ }^{1-4}$ in England before the mid-1950s. Although Lighthill is still active in the field, 1 relatively recent advances in the jet noise theory have been contributed by Powell, 5, 6 Williams, ${ }^{7,8}$ and others. Most experimental investigators have satisfied themselves with varying such gross parameters as jet diameter, 9,10 velocity, ${ }^{11-13}$ and density ${ }^{13}$ while measuring the spectral content, direction, and magnitude of the radiated sound. From these investigations an overall picture of exhaust jet noise generation has emerged, which is in good qualitative agreement with observed noise levels and directivity. Relatively few investigators have addressed themselves to actual experimental research on the details of the jet exhaust mixing process that gives rise to the sound field. ${ }^{14-18}$

Noise measurements in the far field show that, from a noise-generation point of view, the jet is analogous to a train of convected quadrupoles traveling at a speed roughly 0.6 of the core velocity, that is, at a velocity very close to that observed at the middle of a shear layer along a line directly downstream from the lip of the jet. In addition, recent measurements by Davies et al. ${ }^{17}$ have shown that a coherent pressure field having a wavelength that is long, compared with the thickness of the mixing layer, extends from the subsonic jet axis to the near field outside the jet. These pressure disturbances were also measured to travel at about 0.6 of the core velocity throughout the entire region. 
The presence of a coherent pressure wave containing most of its energy at a fixed Strouhal number suggests the generation of the wave in the confined core of the jet by potential flow fluctuations induced by the convection of large intermittent eddies protruding from the mixing region into the core. At low Reynolds numbers, this structure takes the shape of doughnutlike vortex rings propagating away from the jet lip, as shown by the excellent flow visualization of Crow and Champagne. ${ }^{19}$

The research program described in this report seeks a better understanding of the structure of turbulence in the noise-producing region of a round jet. Measurements of turbulence intensities, spectra, and convection velocities were made with a hot wire anemometer in the $1 \frac{1}{2}$-inch-diameter jet shown schematically in Figure 1. A complete description of the apparatus is given in Ref. 20 .

The intent of the current work has been to extend knowledge of jet behavior in the core and the surrounding, spreading, annular mixing region. The work to be described has been primarily experimental, designed to provide a firm foundation for later analysis. The results of these experiments will be used to interpret known characteristics of noise outside the jet as measured by other investigators and to gain a better theoretical understanding of the noise production mechanisms. 
$\omega$

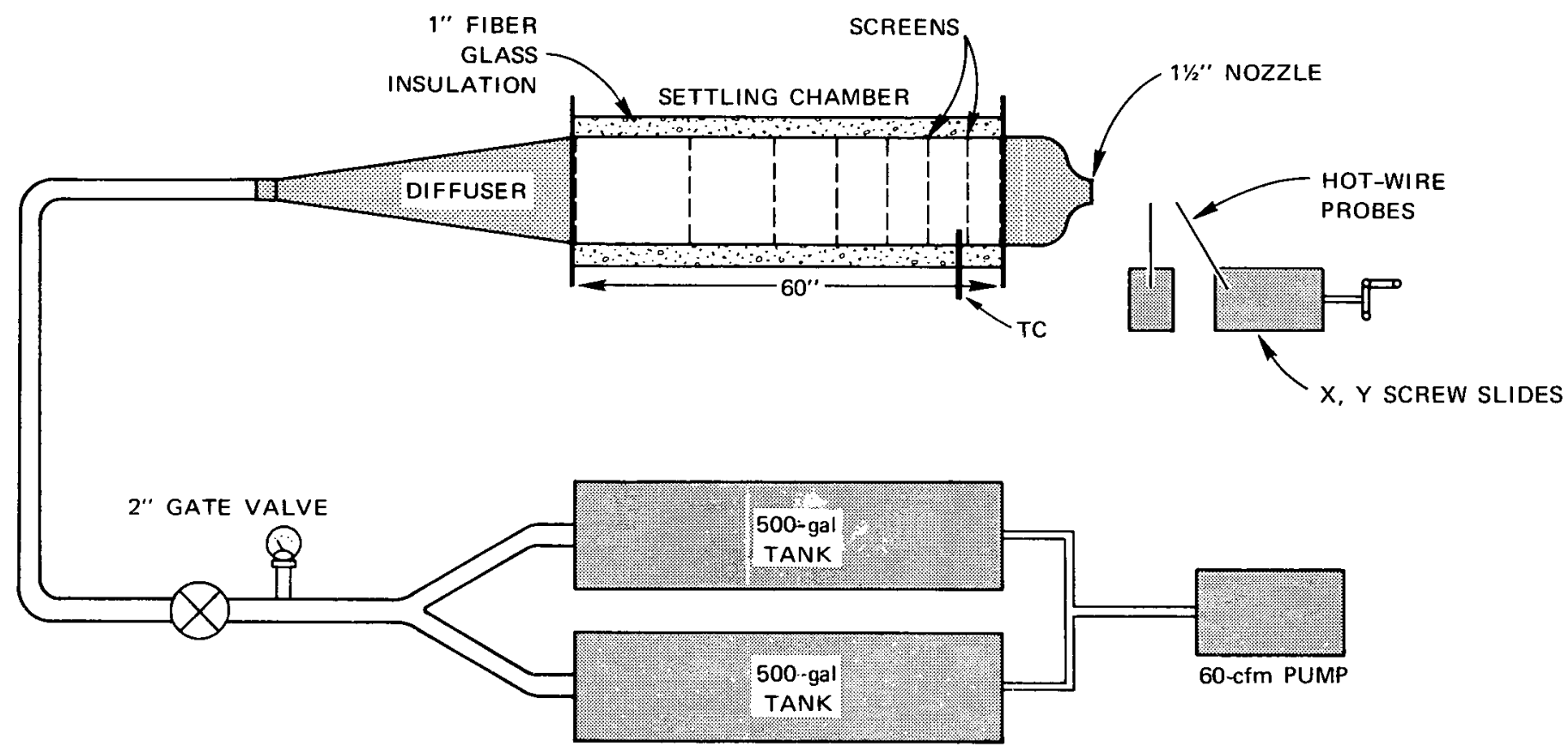

FIGURE 1 SCHEMATIC DIAGRAM OF JET NOISE TEST SYSTEM 


\section{MEAN PROFILE MEASUREMENTS}

The initial characterization of the jet was undertaken during the first year of this contract (see Ref. 20). Additional mean profile measurements were made during the second year to make sure that no serious anomalies were present in the flow. Measurements of mean velocity and turbulence intensity profiles were made using a constant temperature, hot wire anemometer system manufactured by Thermo-Systems Inc. The hot wire probes consisted of 0.2-mil-diameter tungsten wire, $\frac{3}{32}$ inch 1ong, mounted on needle tips. The needles, $\frac{1}{2}$ inch long, were secured by $5 \frac{1}{4} x \frac{1}{4}$-inch, heavy wall, stainless steel tubing. The probe units were attached to a vibrationdampened mount connected to a heavy steel frame. (See Ref. 20 for details.) The bridge output for the mean voltage was filtered through a direct galvanometer connected to a visicorder. For the fluctuating voltage component, a signal conditioner was incorporated. The signal conditioner provided gains of 1, 10, or 100 with a band-pass filter of $5 \mathrm{~Hz}$ to $50 \mathrm{kHz}$. The output of the conditioner was fed into a two-stage operational amplifier consisting of a logarithmic amplifier and an rms meter amplifier having a time constant of $1 \mathrm{sec}$.

Mean velocity profiles taken previously at three Mach numbers and one longitudinal position are shown in Figure 2 , which is reproduced from the work described in Ref. 20. Good agreement between the three cases was obtained. Figure 3 shows the newly measured axial distribution of the mean velocity in both the core $(\mathrm{Y} / \mathrm{D}=0)$ and the annular turbulent region opposite the lip of the jet $(Y / D=0.5)$ at a Mach number of 0.3 . The mean velocity in the core began to decrease at a value of $\mathrm{X} / \mathrm{D}$ between 5 and 6 , while the mean velocity opposite the lip remained constant at least to an 


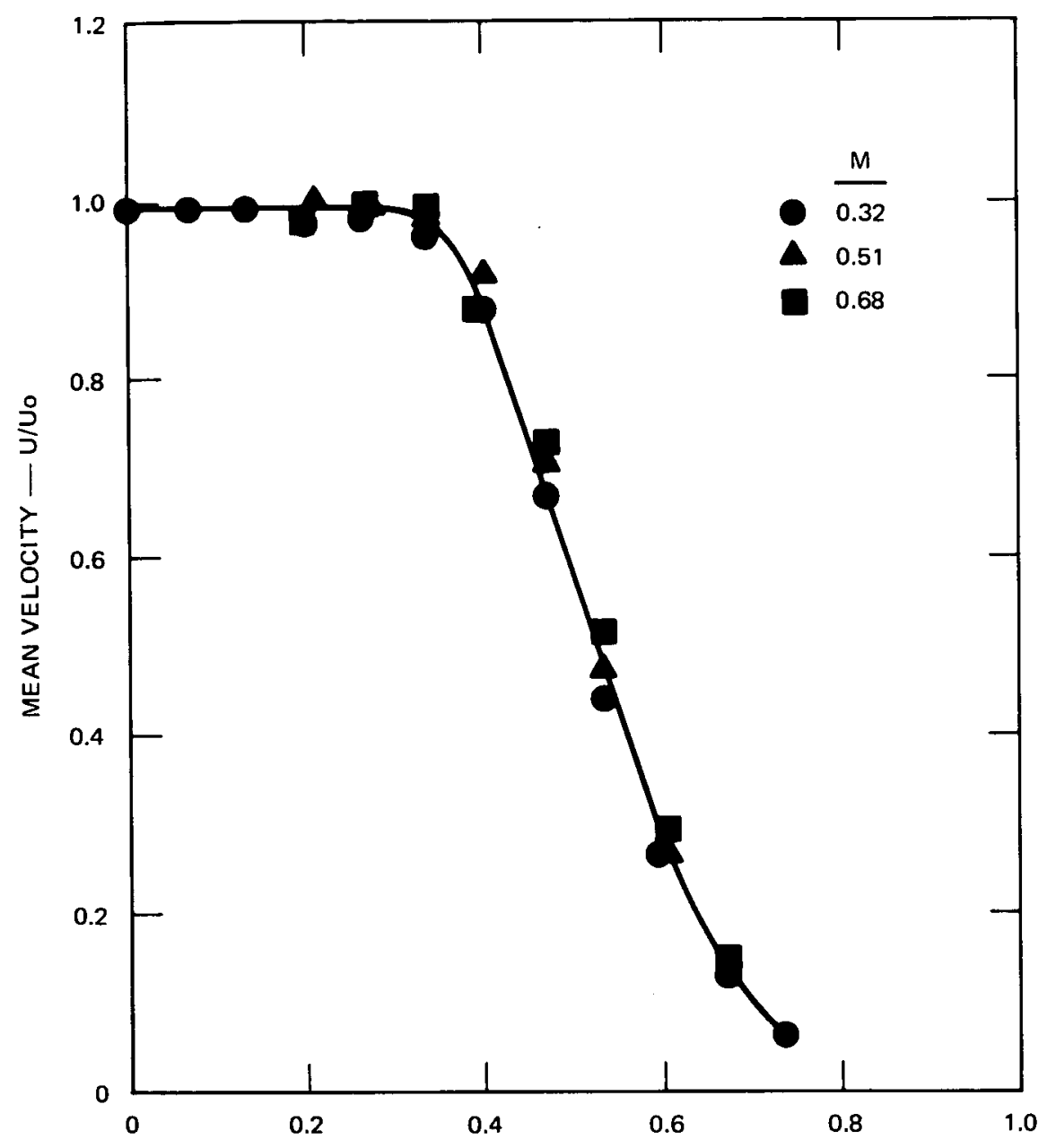

FIGURE 2 MEAN VELOCITY PROFILES AT VARIOUS MACH NUMBERS AT $X / D=2$

axial distance of $\mathrm{X} / \mathrm{D}=10$. Between $\mathrm{X} / \mathrm{D}=5$ and $\mathrm{X} / \mathrm{D}=10$ there is a complicated readjustment in the flow as the profile changes from that of an annular mixing region toward that of a self-similar region downstream. As the center line velocity approaches the lip value, of course, both must begin to decay.

The profiles of mean velocity and turbulence intensity measured very close to the jet exit are shown in Figure 4. The maximum intensity occurred at the point where the mean velocity gradient was maximum, as 


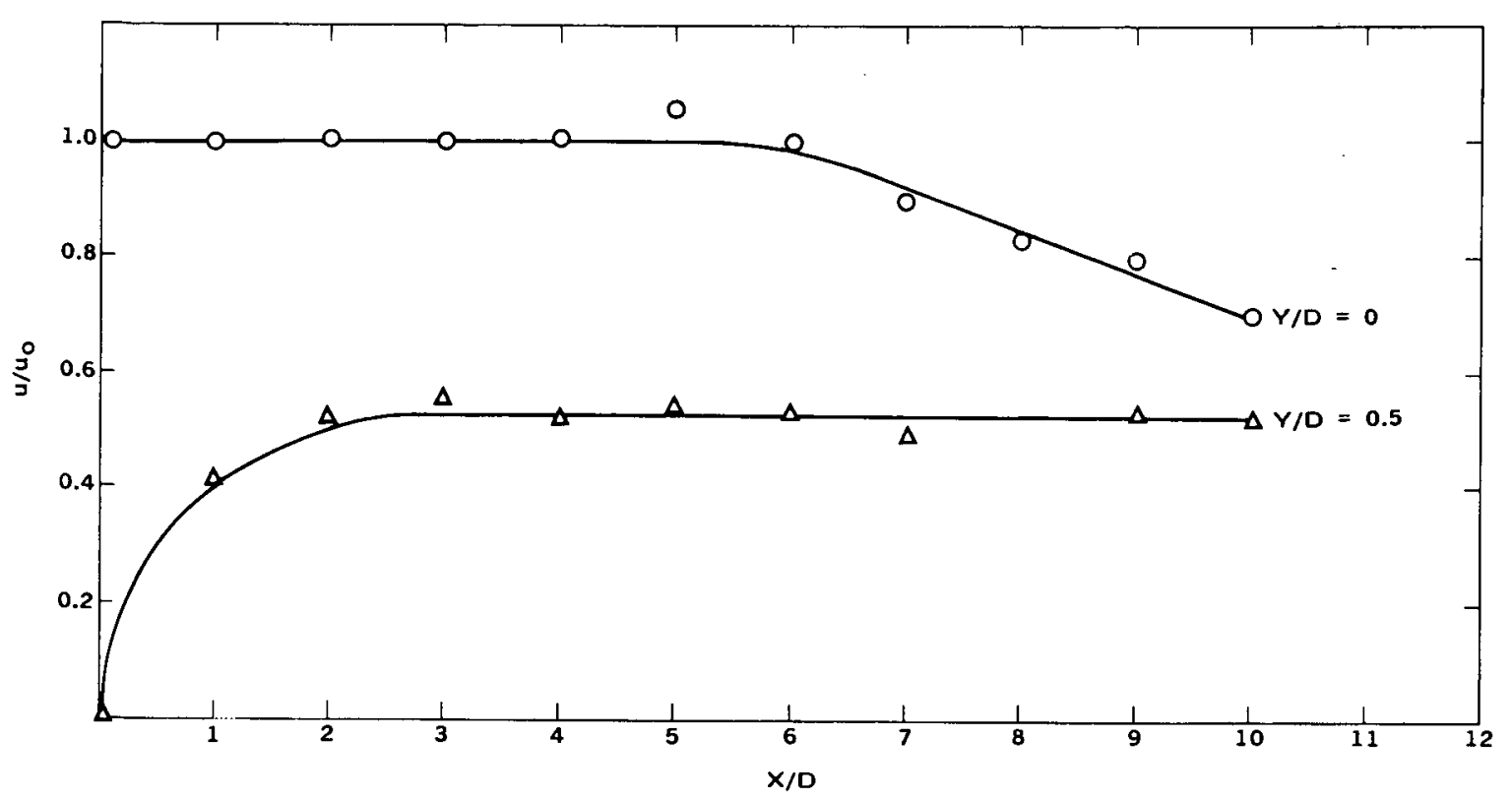

FIGURE 3 VARIATION OF MEAN VELOCITY WITH AXI AL DISTANCE, $M=0.3$

expected. However, the mean velocity profile shows a small peak at $\mathrm{Y} / \mathrm{D}=0.3$ and the turbulence intensity profile a corresponding small peak at $\mathrm{Y} / \mathrm{D}=0.35$. These peaks were not measurable at $X / D=2$ (see Ref. 20), especially for a Mach number of 0.3 , and it was decided to proceed on to the spectrum and correlation measurements, restricting most of the data to $M=0.3$ because this Mach number has been used most often by previous investigators.

Figure 5 shows that the constancy of the mean velocity profile downstream of the lip is reflected in the intensity distribution, which also remains constant after an initial rise in the first two diameters downstream of the nozzle. Also shown is the intensity measured in the core as a function of $\mathrm{X} / \mathrm{D}$. Note that the core intensity increases smoothly from a low value at the exit to a peak value at an $X / D$ of about 9 , with no sudden jump appearing at the end of the core region. The shape of this 


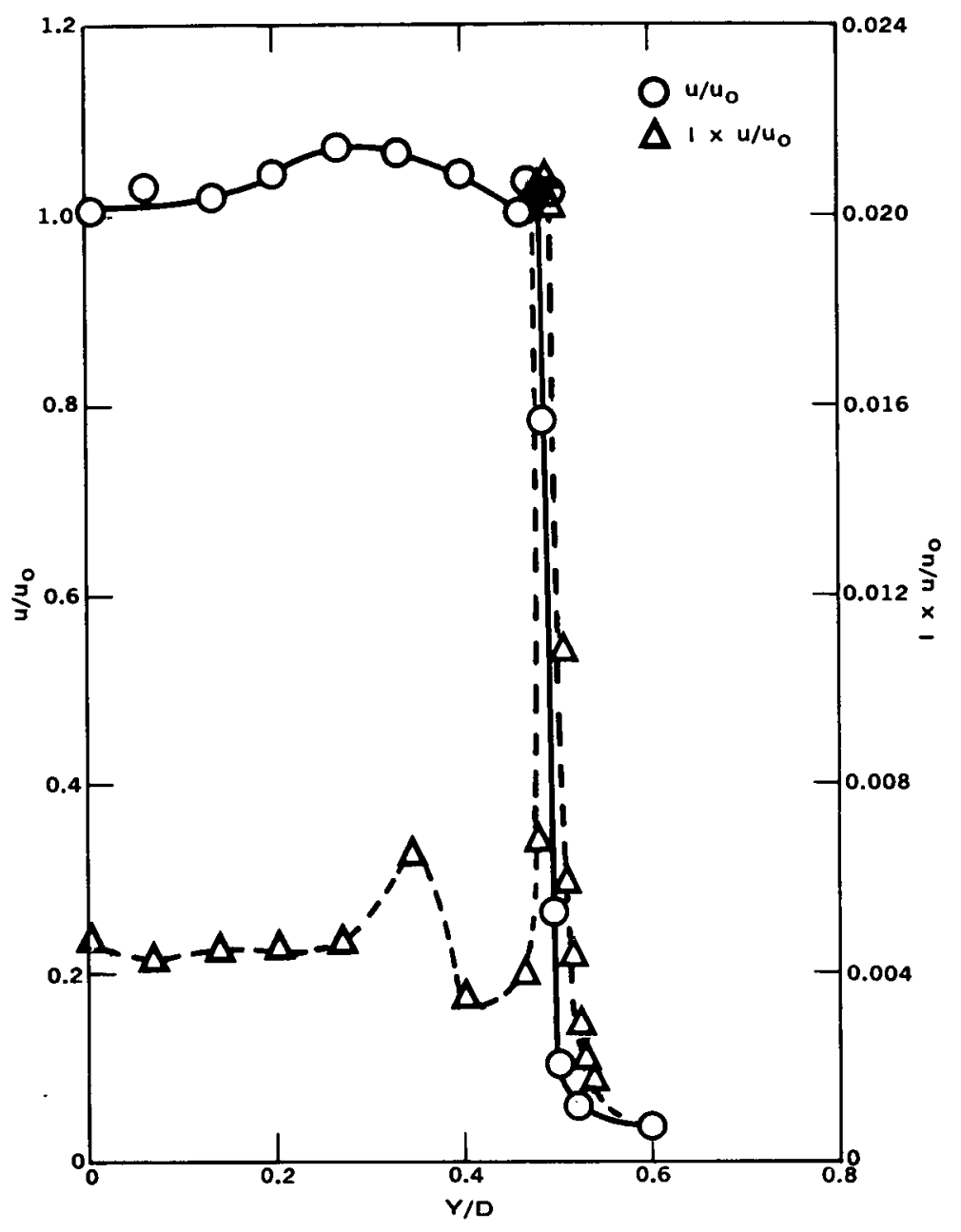

FIGURE 4 TURBULENCE INTENSITY AND MEAN VELOCI TY PROFILES AT JET EXIT, $M=0.3, X / D=0.042$

curve is similar to the axial distribution of impact pressure fluctuations measured by Nagamatsu et al. ${ }^{21}$ Figure 3 also shows why the acoustic power per unit length radiated from the core region of a subsonic jet is essentially constant--because the turbulent intensity in the annular region around the core is constant. 


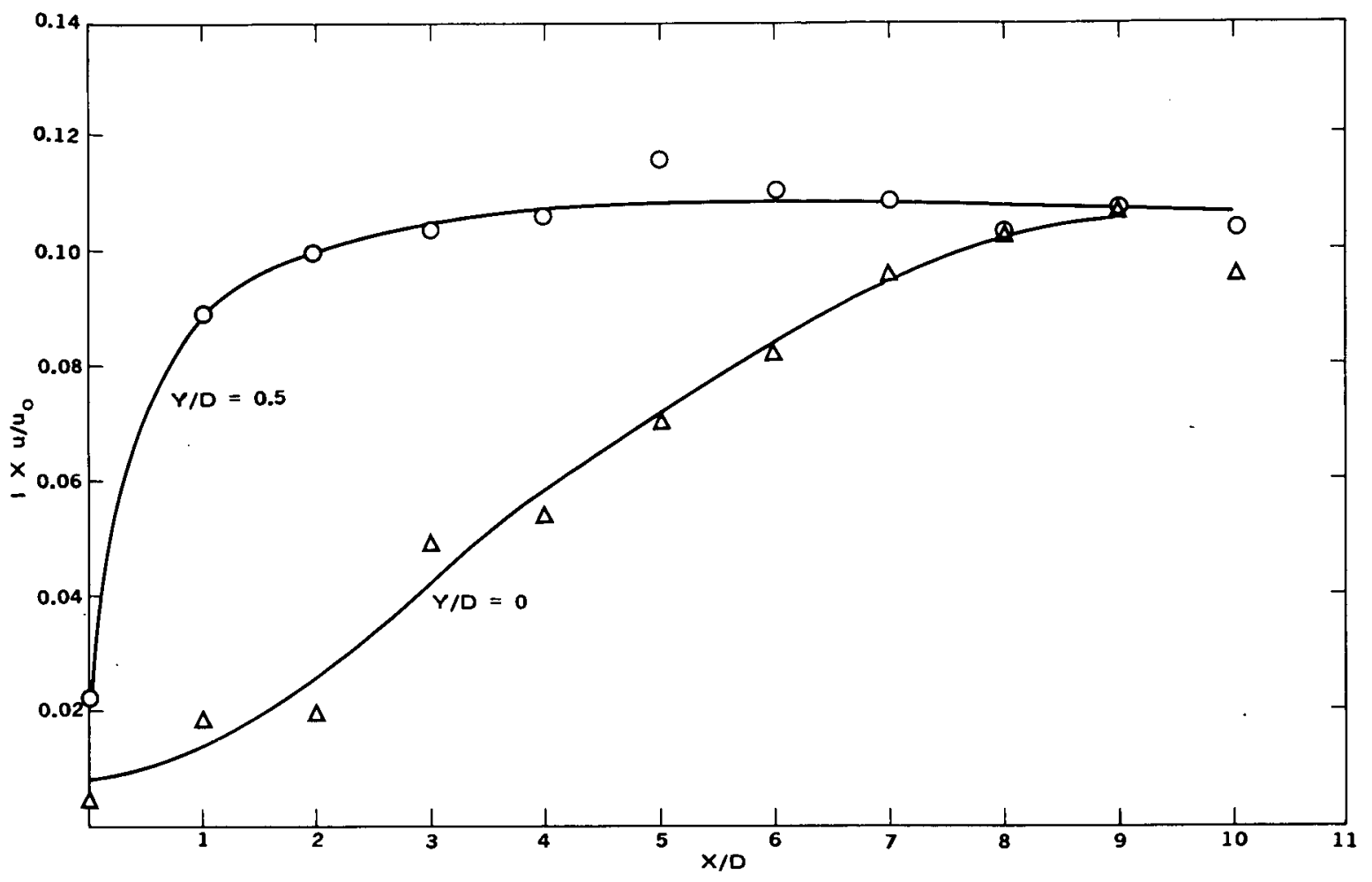

FIGURE 5 VARIATION OF TURBULENCE INTENSITY WITH AXIAL DISTANCE, $M=0.3$ 


\section{PRECTDONG PAGE BLANR NOT FILMED}

\section{POWER SPECTRAL DENSITY MEASUREMENTS}

The power spectral density of the axial velocity fluctuations observed by each hot wire was measured to examine the spectral distribution of the energy in the jet and to ensure that no anomalous signals were present. Davies et al. ${ }^{16}$ reported spectral measurements in the shear region of a 1-inch-diameter jet. Their results indicate that the spectra at $\mathrm{X} / \mathrm{D}=1.5$ do not change significantly with radial position and that at an axial position of $X / D=4.5$ there is a systematic shift of energy to lower frequency with increasing radius. They reason from this that the convection velocity of the eddy pattern varies much less rapidly with radius than does the mean velocity, a conclusion which they further substantiate by direct convection velocity measurements. More recently still, Davies et al. 17 reported more complete spectra measured at various radial positions for $X / D=3$ in a 1-inch-diameter jet. The results they obtained are compared with, and show close agreement with, measurements in the SRI jet. ${ }^{20}$ The shape of the two spectra correspond closely, indicating good agreement between the two sets of data.

Figure 6 shows typical power spectral densities measured at three axial positions in the jet at $M=0.3$. In the core, there are two peaks in the spectra, a broad peak at approximately $1.3 \mathrm{kHz}$ and a narrow, highfrequency peak at approximately $21 \mathrm{kHz}$. The lower frequency peak increases in magnitude with distance from the nozzle exit, while the higher frequency peak decreases in magnitude. The quantitative variation in the magnitude of the $1.3 \mathrm{kHz}$ signal with axial distance from the nozzle exit is shown in Figure 7. The energy of a $10-\mathrm{Hz}$ bandwidth signal at $1.3 \mathrm{kHz}$ increases by 


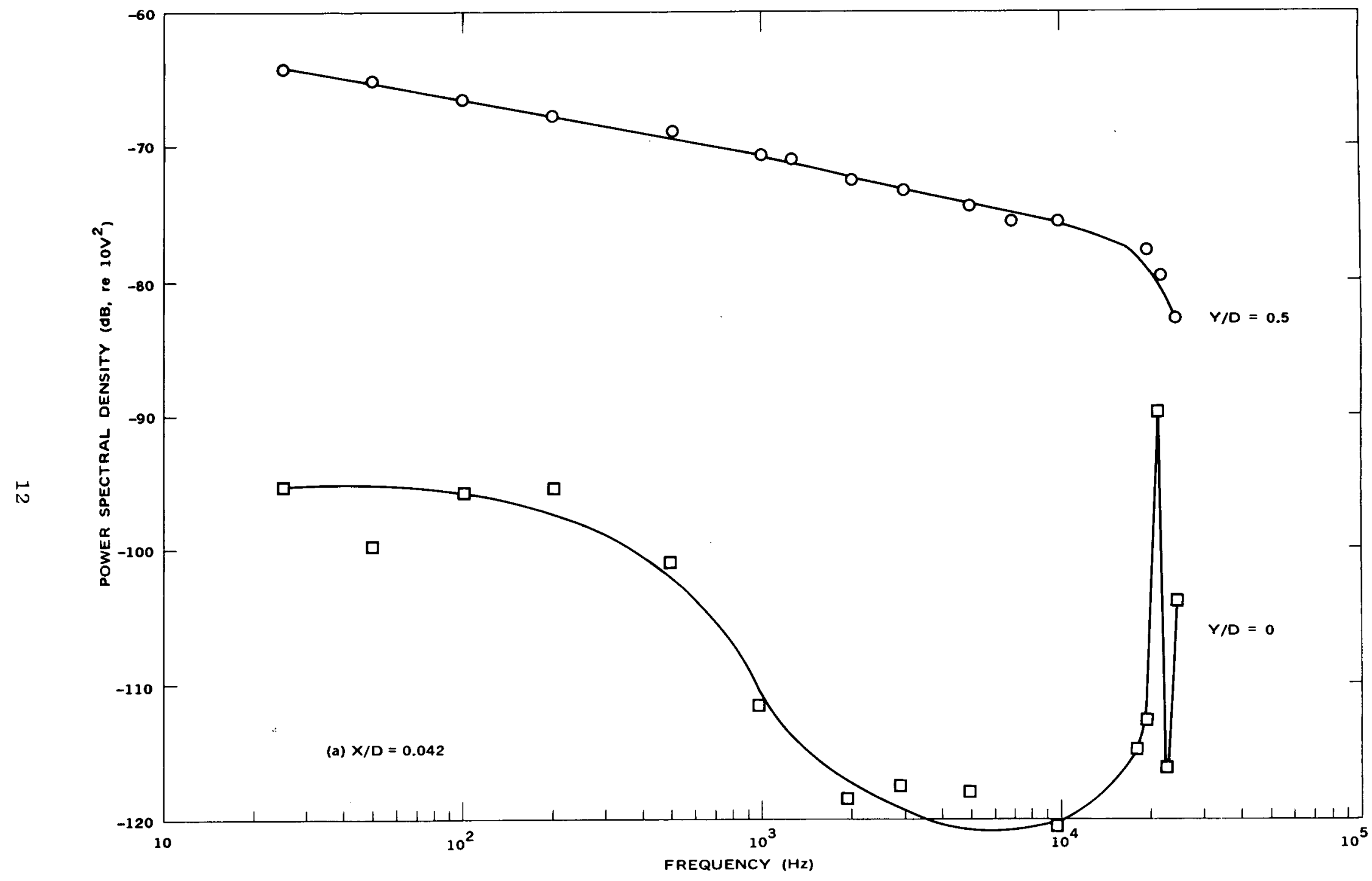

FIGURE 6 POWER SPECTRAL DENSITY AT DIFFERENT RADIAL POSITIONS, $M=0.3$ 


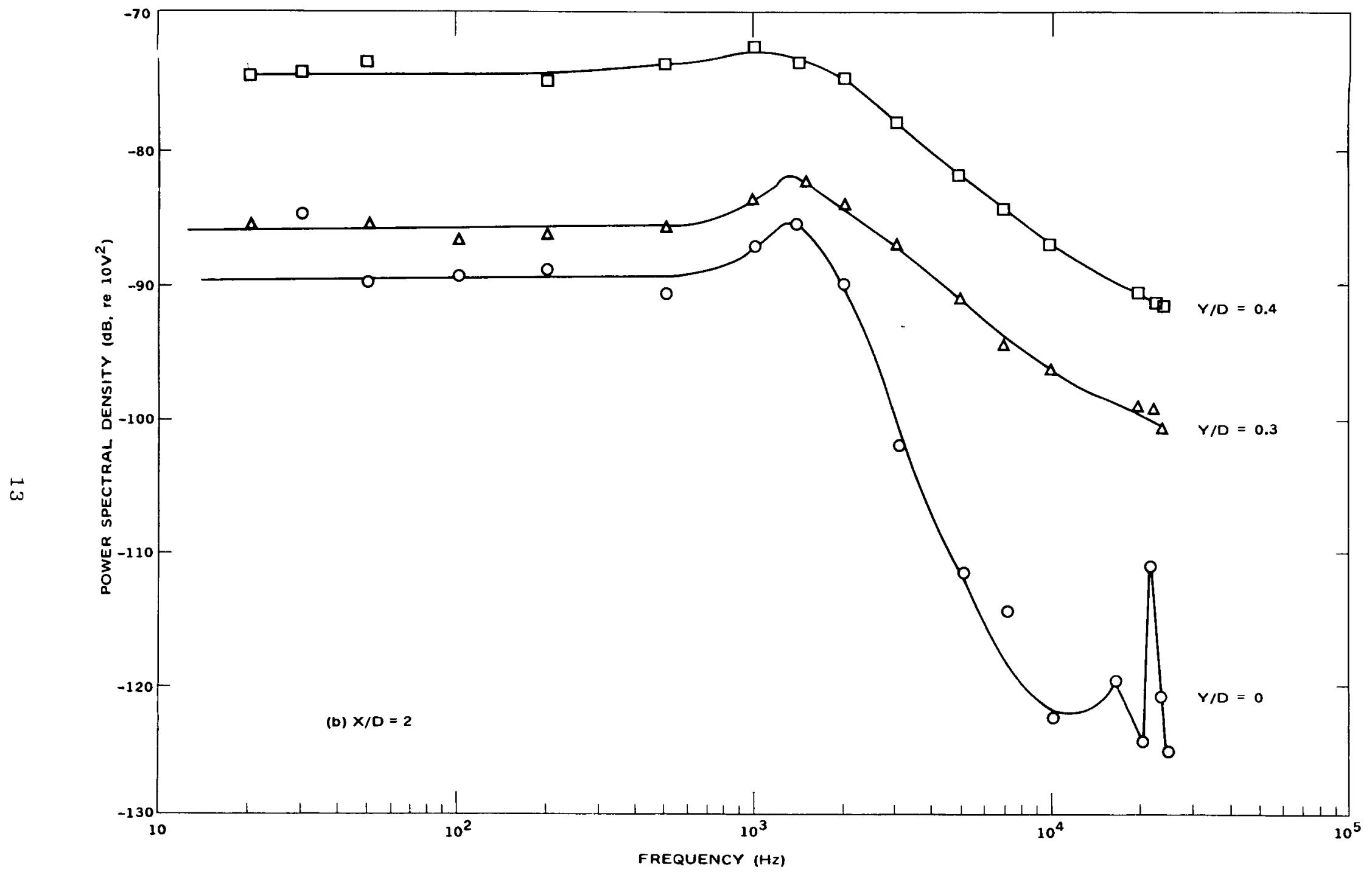

FIGURE 6 (cont inued) 


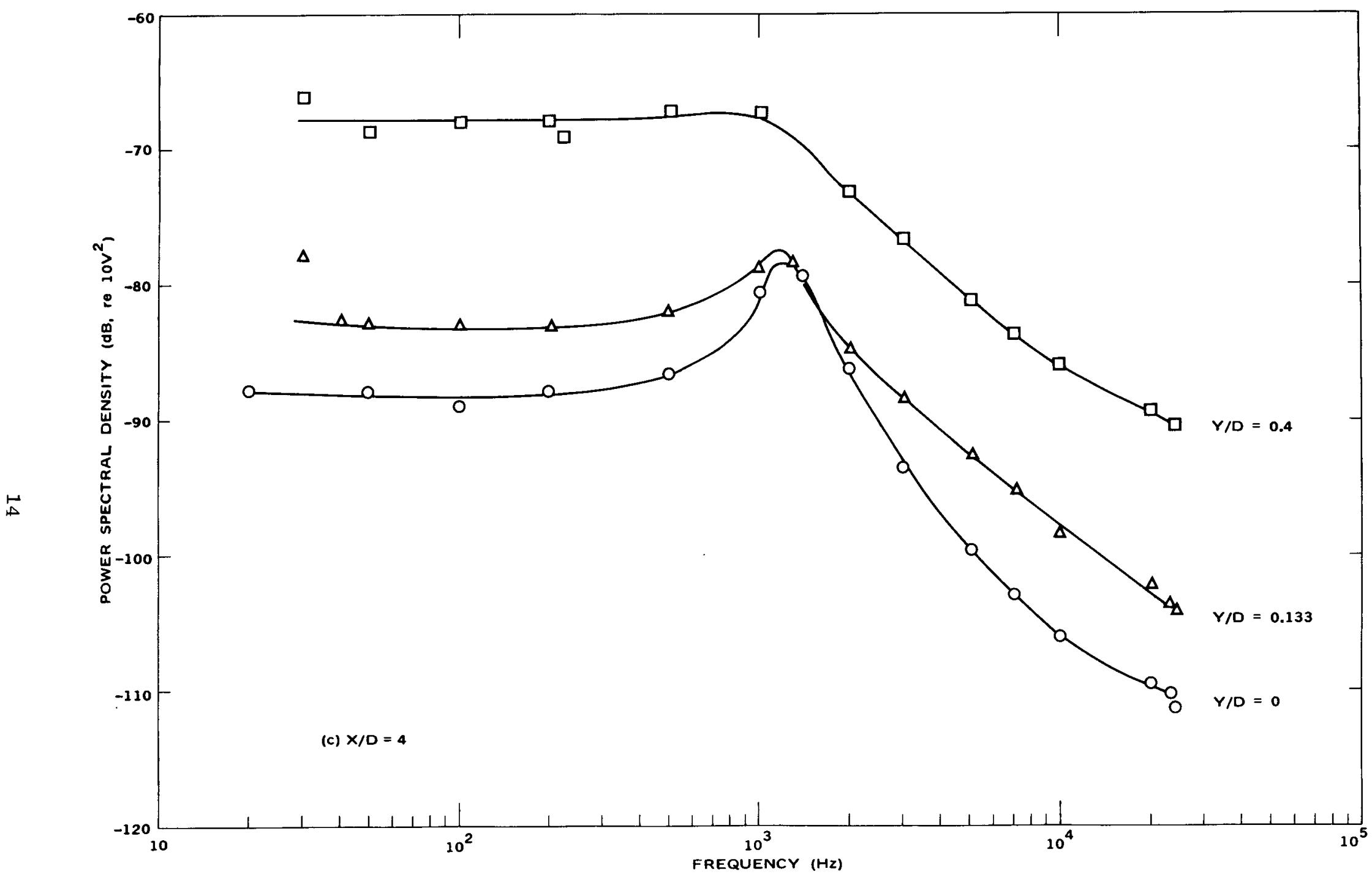

FIGURE 6 (concluded) 


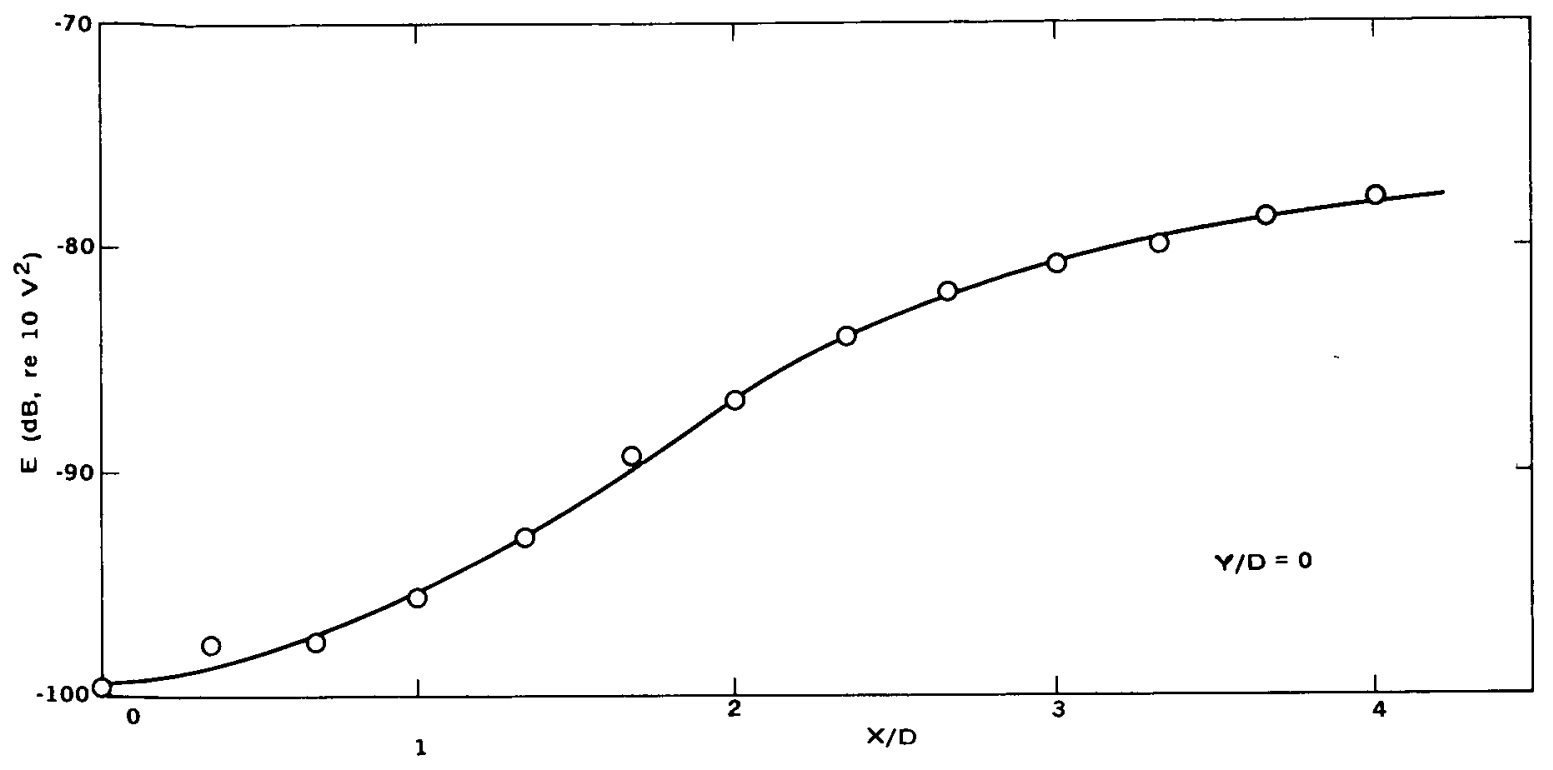

\section{FIGURE 7 VARIATION OF ENERGY IN BAND CENTERED AT $1300 \mathrm{~Hz}$ WITH AXIAL DISTANCE ALONG JET AXIS, $M=0.3$, FILTER BANDWIDTH $=10 \mathrm{~Hz}$}

about $22 \mathrm{~dB}$ (a factor of approximately 12) along the jet center line between the jet exit and the end of the core $(X / D=0.4)$. Neither of these two peaks is observed at the center $(Y / D=0.5)$ of the turbulence mixing region surrounding the jet core, since the intense turbulence there masks these lower energy signals. Note that the power spectra near the center of the mixing region are more uniform than in the core region of the jet and do not exhibit peaked characteristics.

The lower frequency peak observed in the core region of the jet at 1.3 $\mathrm{kHz}$ is approximately at the same peak frequency observed by both Lawrence 14 in a 3.5-inch-diameter jet and Davies et al. ${ }^{17}$ in a 1 -inch-diameter jet. This implies that the peak frequency cannot be nondimensionalized as a Strouhal number using the outlet diameter as the characteristic length. Other evidence available from additional data taken in our laboratory at $M=0.5$ and 0.7 and from the data of Nagamatsu et al. ${ }^{21}$ at $M=0.6$ is that the peak frequency is proportional to the velocity, indicating a constant (within experimental error) characteristic length for subsonic jet flow. One such 
length in the flow is the distance between the intermittent bursts that are observed at the boundary between the core and the turbulent annulus surrounding it. Crow and Champagne 19 have taken excellent photographs of jet flow at low Reynolds numbers which show clearly the intermittent, vortexlike, bursts at the external edge of the mixing layer. These bursts are characteristic of the large-scale turbulence structure (or intermittency) believed to excite the coherent acoustic disturbances in the near field of the jet flow. At higher Reynolds numbers, the details of the intermittent vortexlike structure probably are lost, being replaced by a jetlike structure similar to that observed in the outer region of a turbulent boundary layer. $^{22}$ Experiments are continuing at SRI to determine the characteristic length associated with these phenomena.

Figure 6 also shows the narrow peak at the higher frequency, which is most noticeable in the core. This peak has also been observed by Davies et al. 17 and by Laurence. ${ }^{14}$ In this case, the frequency can be nondimensionalized by the core velocity and the estimated boundary layer displacement thickness, giving a Strouhal number of approximately 0.3 , equal to that for vortex shedding from a cylinder. This idea was suggested by S. J. Kline* of Stanford University, whose associates recently found a similar result for flows of different velocities along the two sides of a flat plate; in that case, the characteristic velocity was the difference between the velocities along the two sides.

The power spectral densities in the core region of the jet at Mach numbers of $0.3,0.5$, and 0.7 are shown in Figure 8 . The lowest frequency peak in each case shifts to higher frequency with increasing Mach number. However, at $M=0.5$ and $M=0.7$, there are additional peaks at higher frequencies. It is suspected that these peaks are due to some anomalous

* Private communication. 


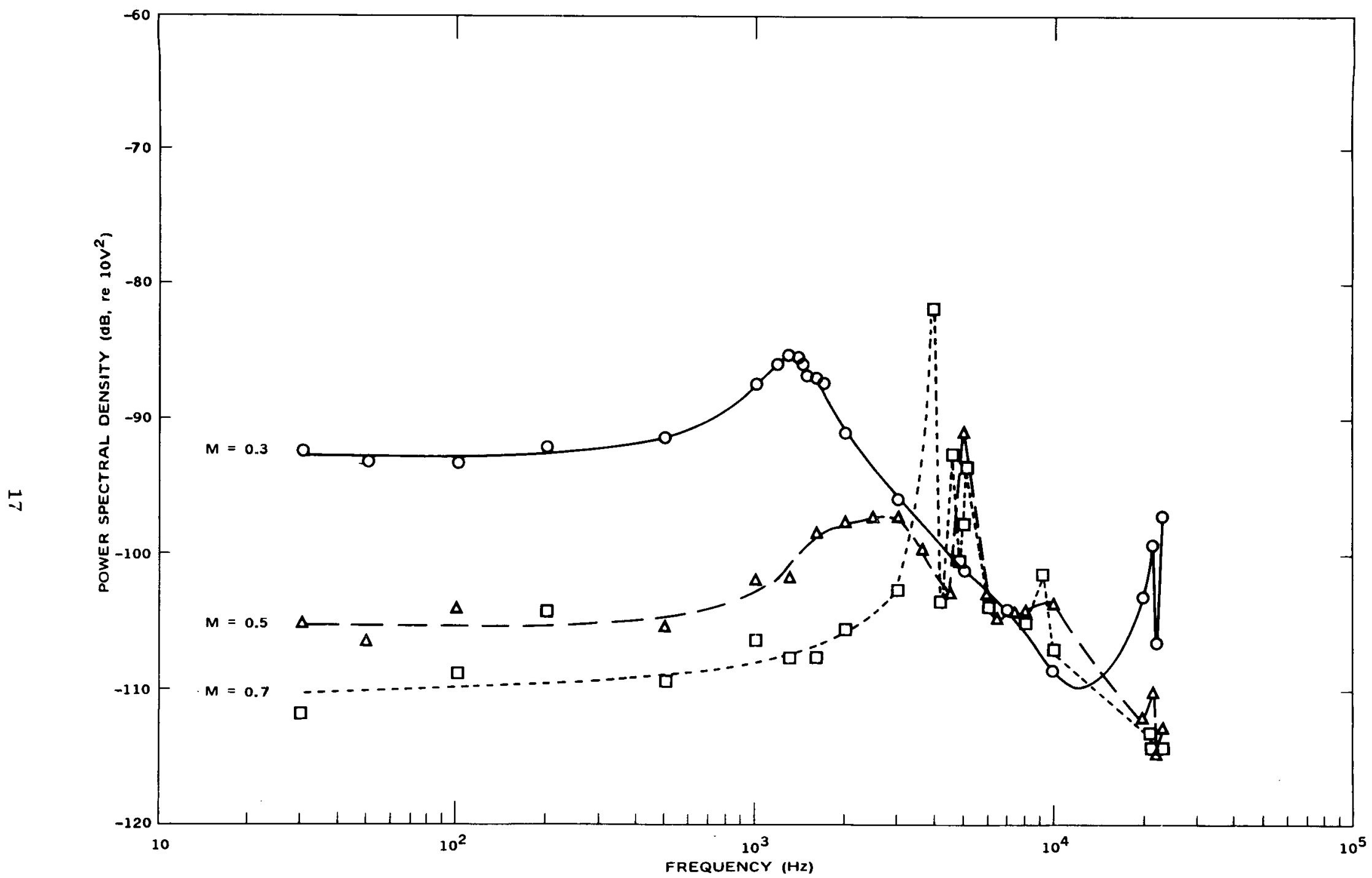

FIGURE 8 POWER SPECTRAL DENSITY AT DIFFERENT MACH NUMBERS, $X / D=2, Y / D=0$ 
flow in the jet. As a preliminary step to looking for the source of this flow, the velocity profile at the exit of the settling chamber with the nozzle removed was measured at $M=0.7$. As shown in Figure 9, there is a slight overshoot at the edge of the wall boundary layer. It is not known if this is an axisymmetric phenomenon. However, there is a similar bulge in the velocity profile and the turbulence intensity at the nozzle exit at $M=0.3$. Because of the low level of the signals measured in the core, an anomaly of this type, although not strong enough to significantly affect the overall jet turbulence structure, is certainly capable of altering the power spectra in the core. Note, for example, that the low frequency spectrum at $M=0.7$ is almost $20 \mathrm{~dB}$ lower than that at $M=0.3$; hence, any anomalous disturbance is more likely to be observed. The source of the high-frequency peak in the jet core spectra at $23 \mathrm{kHz}$ when $\mathrm{M}=0.3$ is believed to be due to the development of the preferred mode of instability of the initial region of the shear layer near the lip of the jet. The amplitude of this signal is highest near the jet exit and decreases with axial distance from the jet exit, a fact that is certainly consistent with the above picture. As mentioned previously, workers at Stanford University under Professor S. J. Kline have found that the two-dimensional shear layer formed by flows of different velocities off either side of a flat plate have a preferred instability mode that scales with the Strouhal number based upon the sum of the boundary-layer displacement thicknesses.

Several experiments were run in the $1 \frac{1}{2}$-inch-diameter jet to determine the behavior of the high-frequency peak with varying nozzle exit conditions. Figure 10a shows spectra for the cases of a high-turbulence nozzle flow and with a boundary-layer trip wire in the nozzle, compared with the standard low-turbulence nozzle. High turbulence was generated by placing a $\frac{1}{4}$-inch mesh by 0.05 -inch-diameter wire screen inside the nozzle $\frac{1}{2}$ inch upstream of the exit. The trip wire was a $\frac{1}{16}$-inch-diameter wire placed adjacent to the nozzle wall $\frac{3}{4}$ inch upstream of the exit. Both of these modifications 


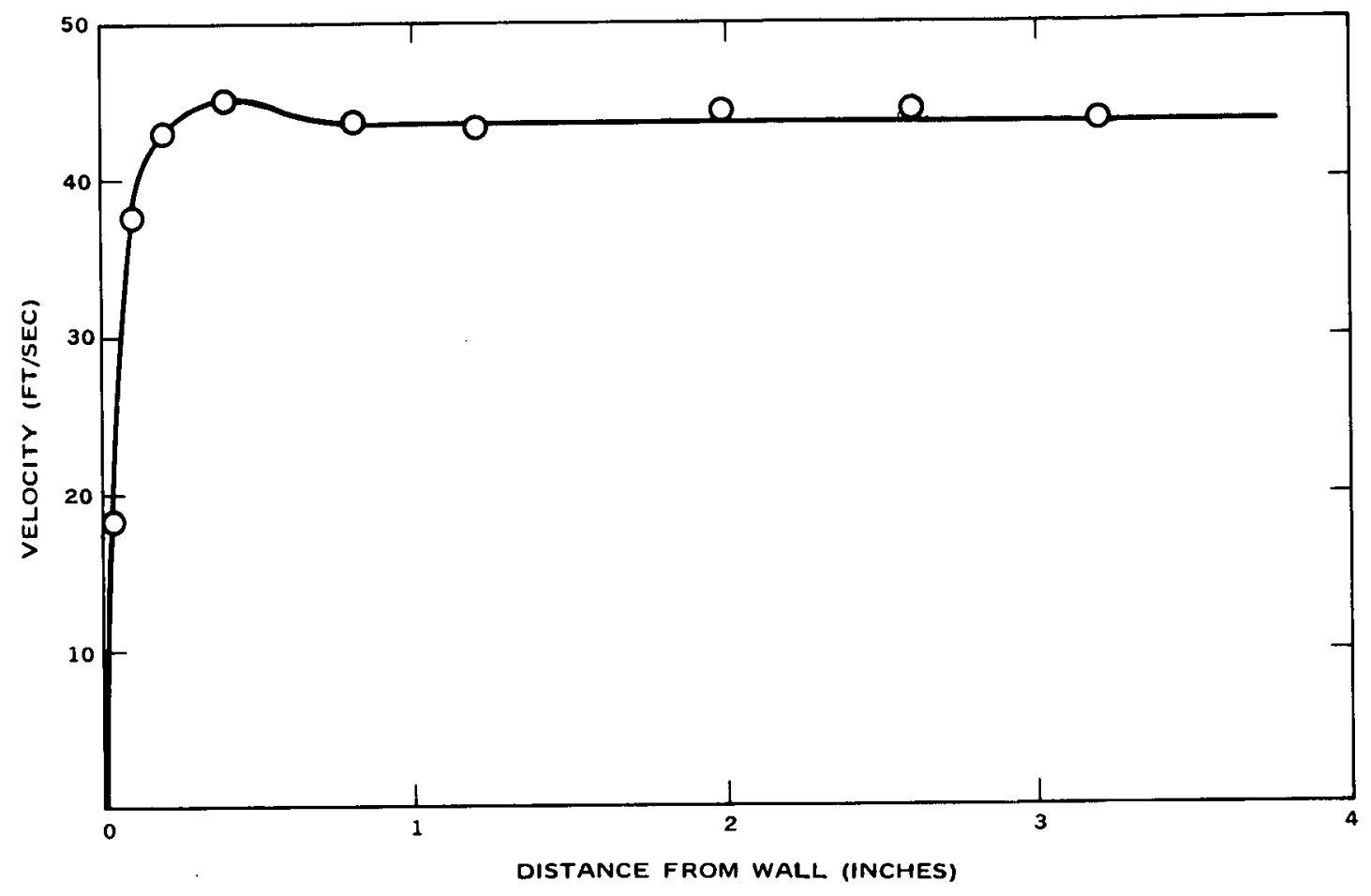

FIGURE 9 VELOCITY PROFILE IN THE SETTLING CHAMBER, $M=0.7$

increased the energy in the jet core at higher frequencies and slightly shifted the low-frequency peak. However, no systematic effect on the higher frequency peak was observed. No firm conclusion should be drawn from these relatively general measurements; rather more detailed measurements of the boundary-layer profile at the jet exit must be obtained to relate the present results' to those of Kline's group.

Spectra for the same nozzle but with a 10-inch pipe extension are shown in Figure 10b. There are peaks in the spectrum at the jet axis at approximately 5,10,15, and $20 \mathrm{kHz}$. This is an interesting observation which shows that the nozzle exit velocity profile certainly changes the peaks in the spectrum. Further interpretation must await detailed measurements of velocity and turbulence intensity profiles at the exit of the nozzle extension. 


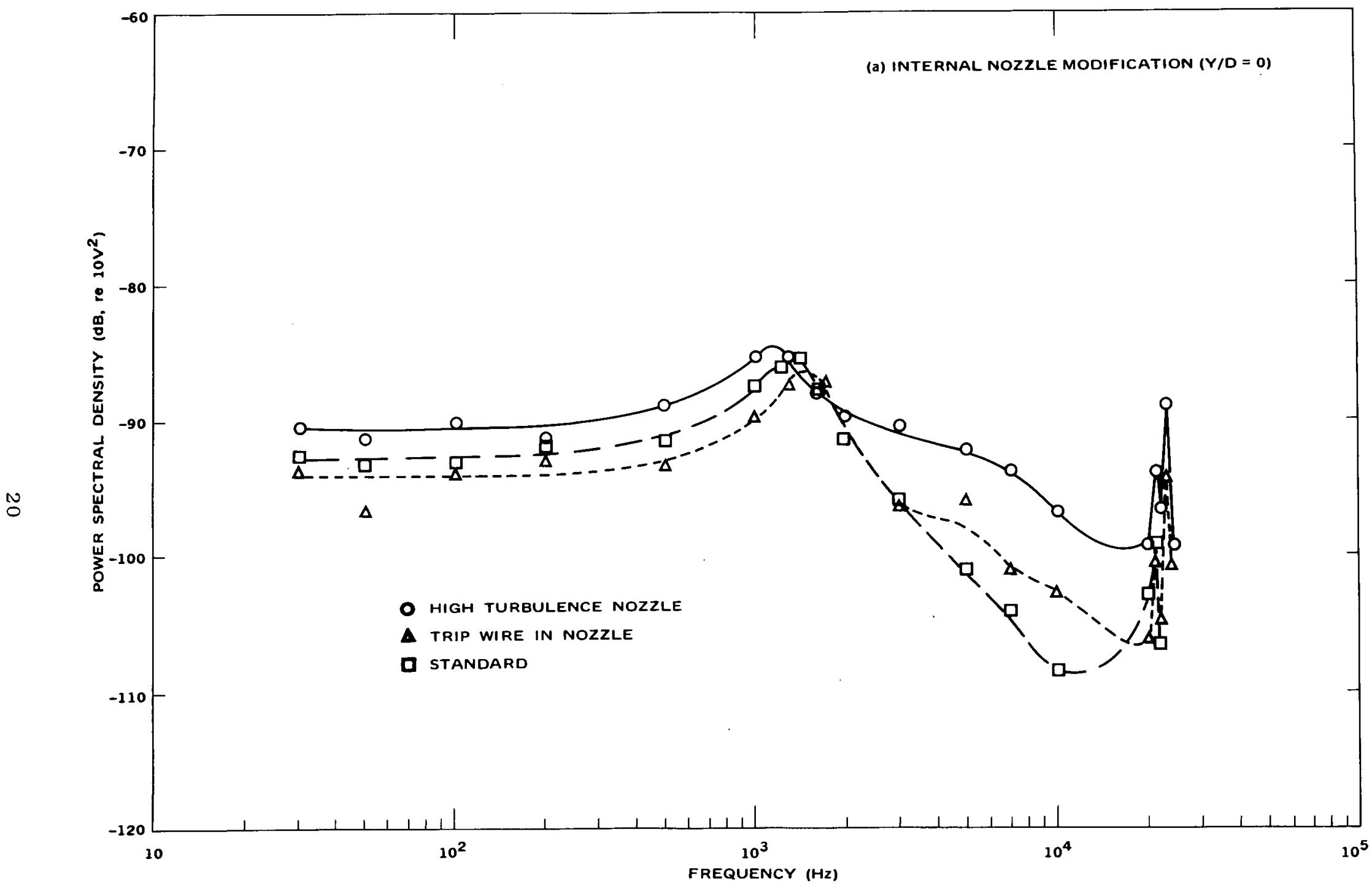

FIGURE 10 POWER SPECTRAL DENSITIES WITH MODIFIED NOZZLES, $X / D=2, M=0.3$ 


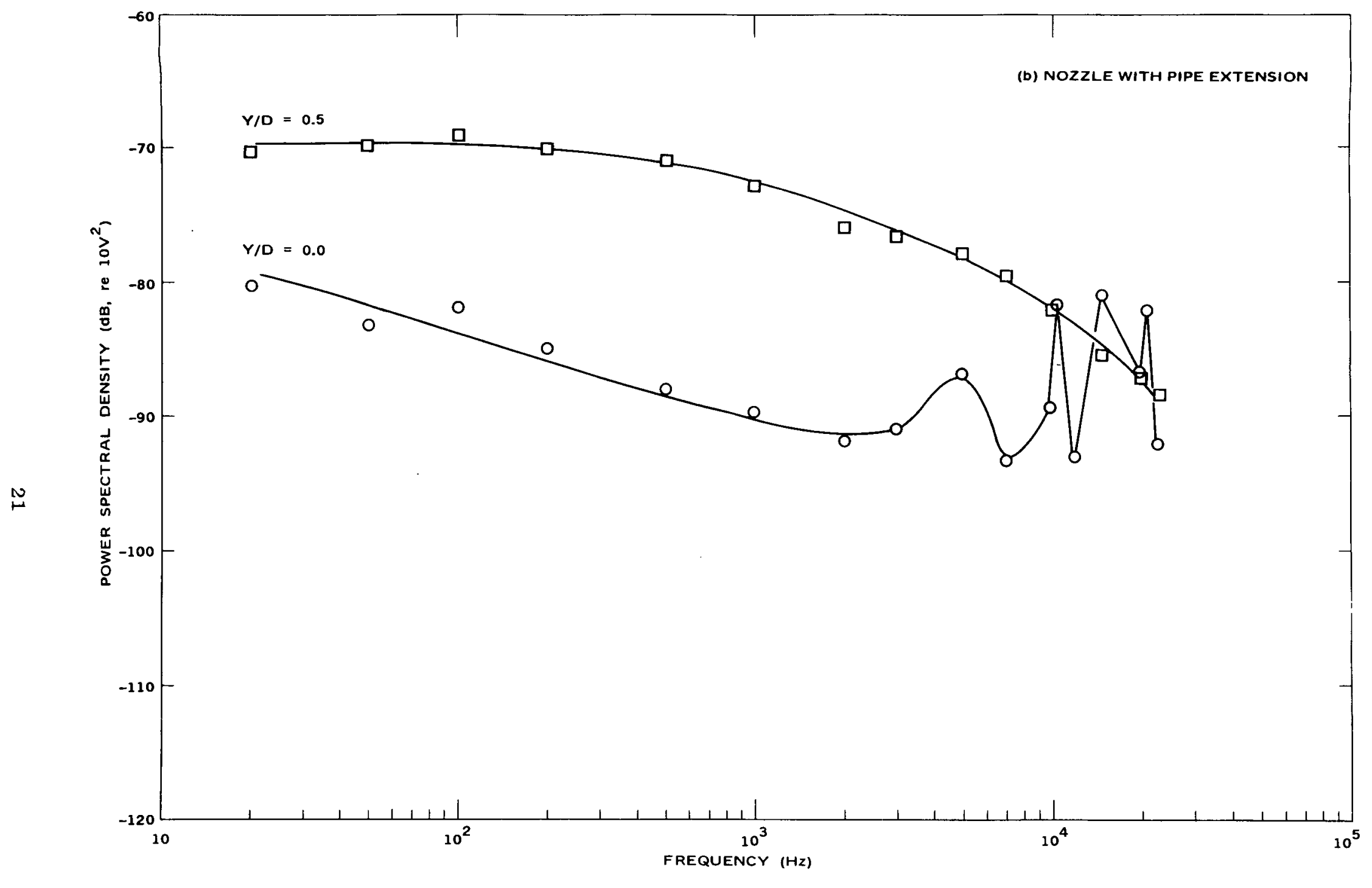

FIGURE 10 (Concluded) 


\section{CROSS-CORRELATION MEASUREMENTS}

Measurements using two hot wire probes, from which auto-correlations and axial cross-correlations (used to compute convection velocities) were computed, were carried out for low core turbulence levels only. For numerical computations, the data were first converted from analog to digital form on an SDS 930 computer in segments of $102.4 \mathrm{msec}$ duration. The digital data were then analyzed on a $\mathrm{CDC} 6400$ computer, using a spectral analysis program developed by Singleton and Poulter for single-channel data but extended to two-channel data. The data were read into the computer, and the two input signals were then plotted as a visual check on the analog-to-digital conversion process. All plots were made on line, using a CDC 280 microfilm plotter.

An example of the input data is shown in Figure 11. Channel A is the upstream wire of the pair. The vertical scale is an arbitrary binary scale chosen for convenience. A rough calibration was made, however, by analyzing a $1 \mathrm{kHz}$, lv rms signal, which indicated that each unit on the vertical corresponded to approximately $22 \mathrm{mv}$. A rigorous calibration was not carried out since absolute magnitudes do not enter the correlation measurements. At the location inside the lip $(Y / D=0.4$, Figure 11 a) there is a definite skewness of the signal in the negative direction while at the location outside the lip ( $Y / D=0.667$, Figure $11 \mathrm{~b})$ the signal is much more symmetric. A pulse in the negative direction represents a decrease in velocity, as though jets containing lower velocity fluid from the center of the mixing region are impinging on the turbulence near the boundary of the core. Future work will include a more thorough study of these data in an attempt to understand this phenomenon. 


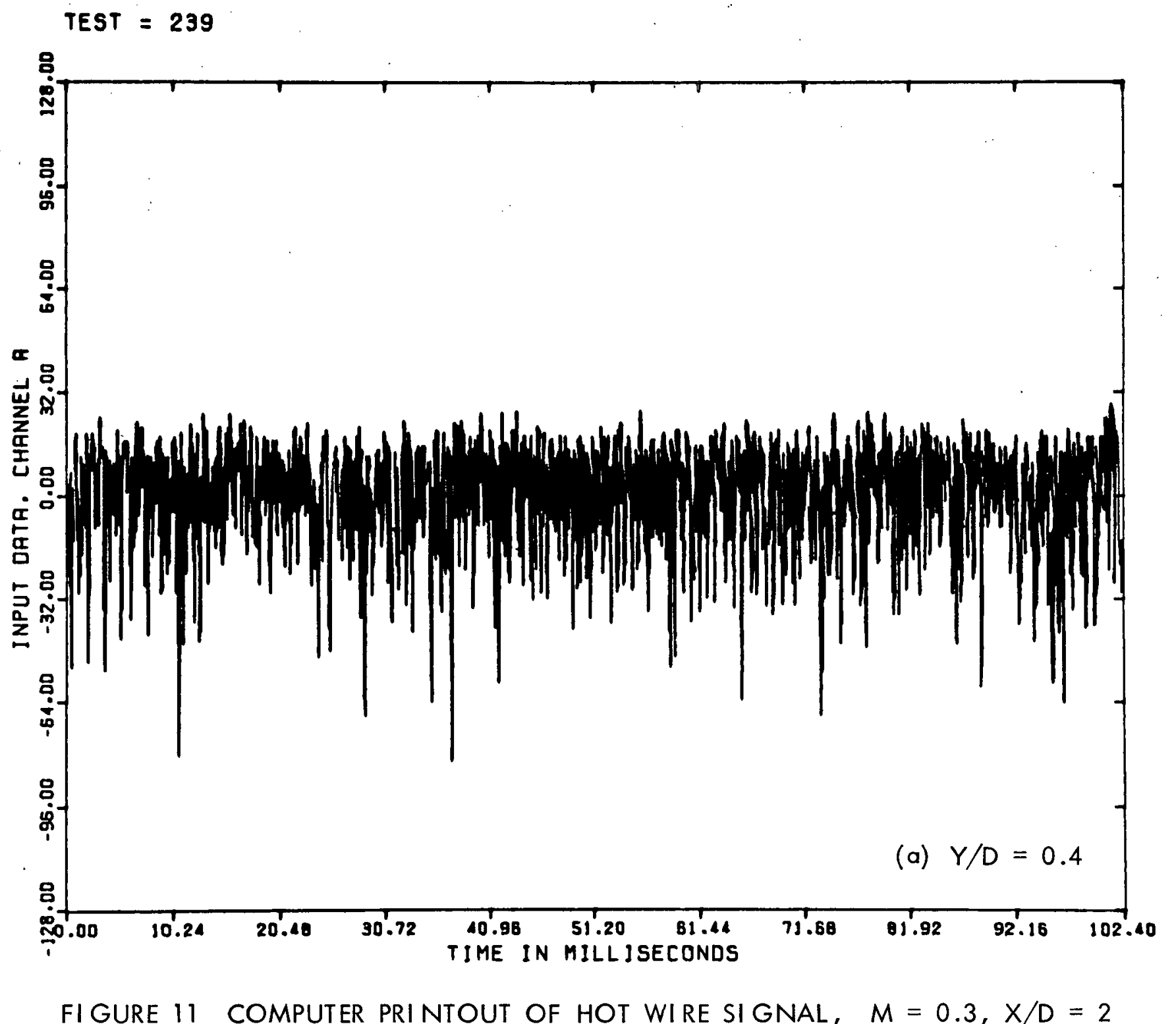




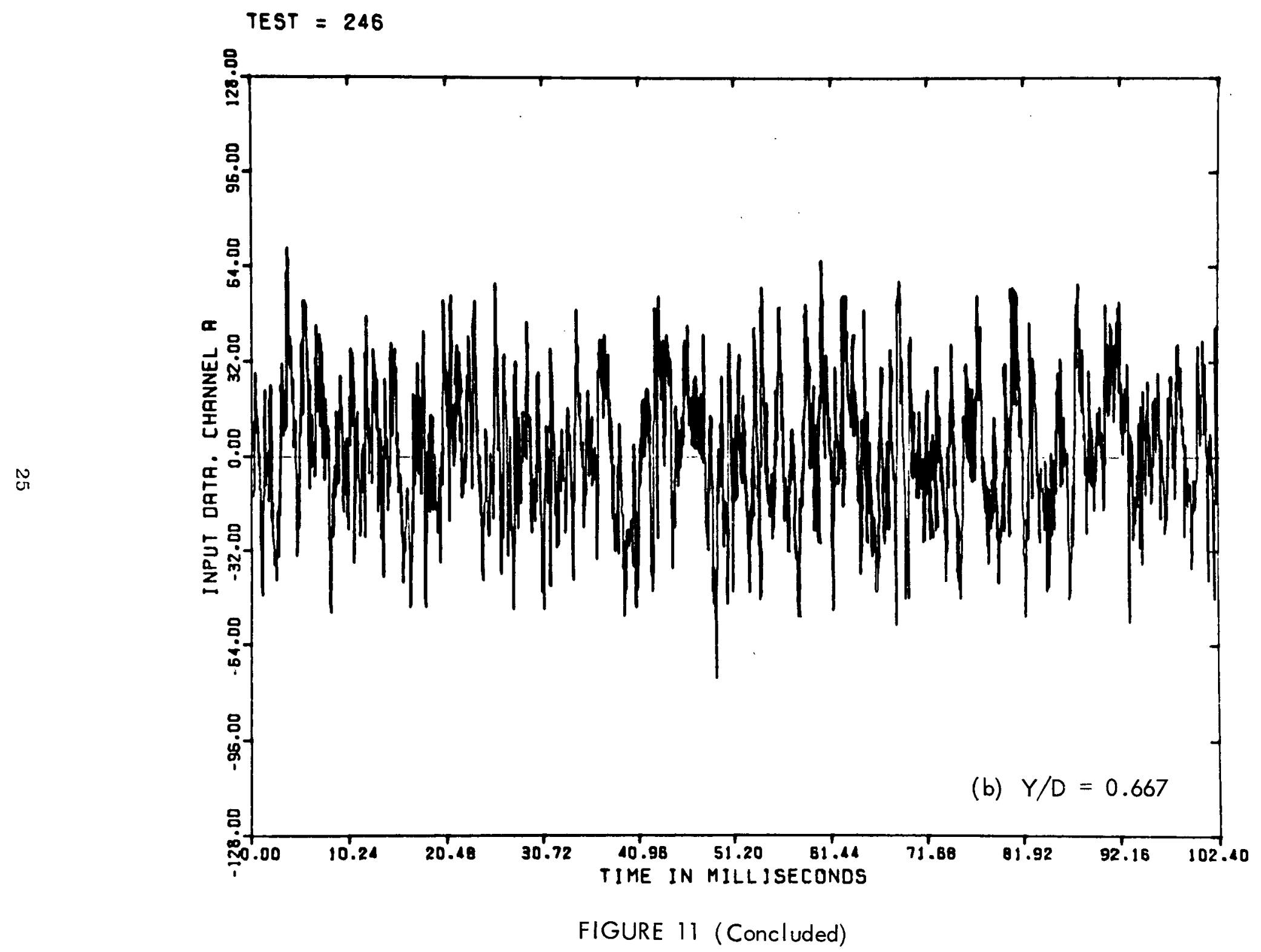


An example of the digital spectrum in the core at $M=0.3$, recovered from the digitized data, is shown in Figure 12. This spectrum is similar in shape to the core spectrum shown in Figure $6 \mathrm{~b}$, with the exception that the high-frequency peak is folded about the cutoff frequency of 11 octaves, which corresponds to $20 \mathrm{kc}$. Because this frequency is well above the center frequency of the highest pass-band studied, and because the total energy contained in the high-frequency peak is small, no attention was paid to this folding.

To calculate correlations, the data on each channel were multiplied by the data window function

$$
\sin ^{2}(\pi j / N) \text { for } j=0,1, \ldots N-1
$$

to reduce interaction of spectral estimates.

The windowed data were then transformed to the frequency domain, using a fast Fourier transform subroutine, and the auto- and cross-spectra computed. These results were then transformed back to the time domain to yield the auto- and cross-correlation functions. For the auto-correlation functions, the lags corresponding to the first zero crossings were interpolated and listed on the plots. For the cross-correlation functions, a search was made for the largest peak, and the 3-point interpolated value and corresponding lag were listed in the plot.

The results were then transformed back to the frequency domain, and an additional step introduced to reduce core storage requirements for intermediate results. The auto- and cross-spectra were scaled for unity power in each channel, then smoothed to gain statistical stability--once with 3-point triangular smoothing and then twice with 15-point triangular smoothing . 


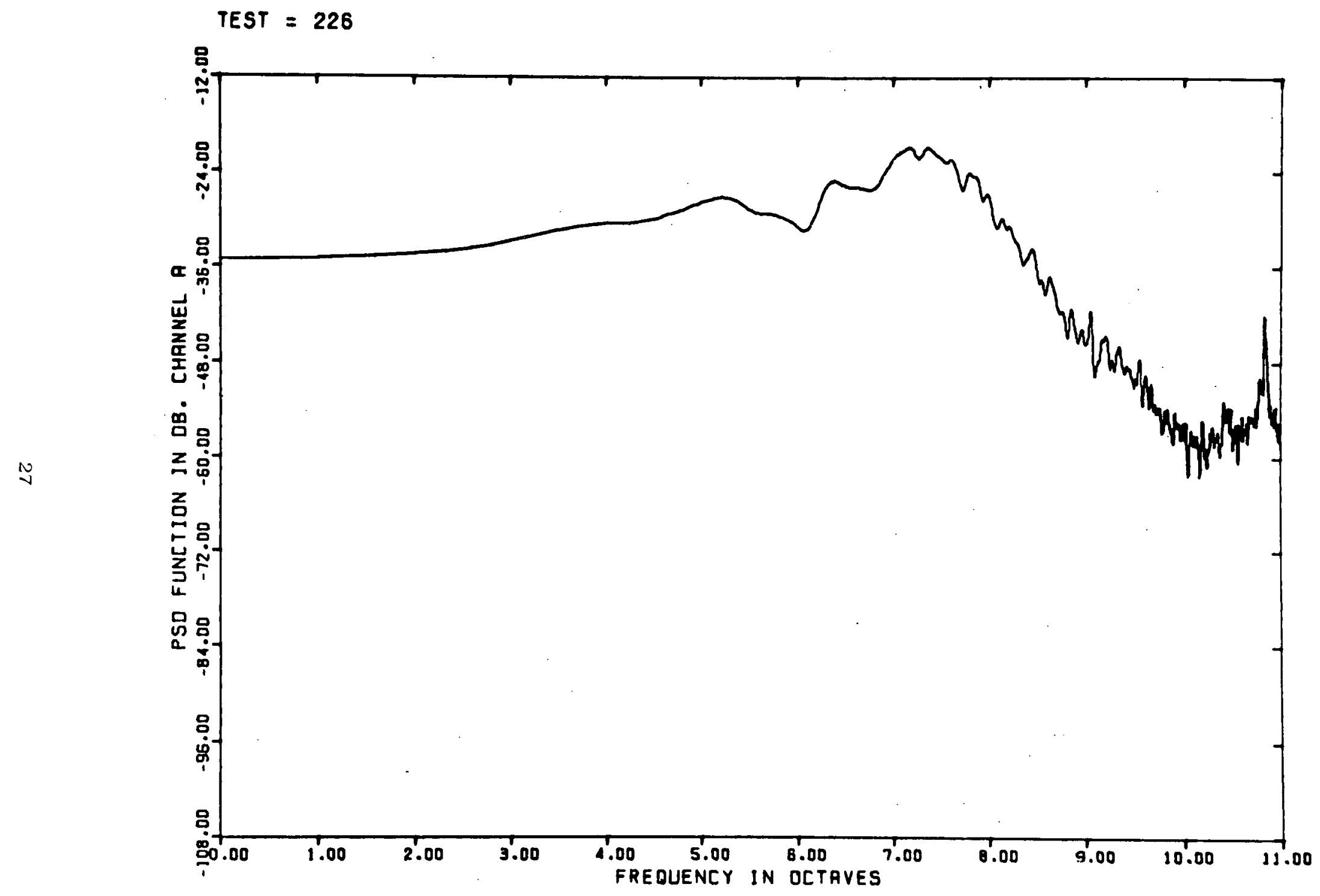

FIGURE 12 COMPUTER PRINTOUT OF POWER SPECTRAL DENSITY, $M=0.3, X / D=2, Y / D=0.133$ 
The computed cross-correlation function, $R_{11}$, between the signals from the two channels was plotted for each test. The 3-point interpolation of the maximum value of the cross-correlation and the corresponding time lag were listed at the top of the plot. The 3-point interpolation was made by taking the maximum value and one point on each side of the maximum and fitting these three points with a quadratic curve. An example of a computed cross-correlation is shown in Figure 13 for two values of wire spacing, to show how the peak values decrease and the associated time lags increase with increased wire spacing. In addition to curves like the one shown in Figure 13, the maximum value of the cross-correlation and five points on either side of the maximum, together with the corresponding time lags, were printed out along with the interpolated values for each test. These data were used to prepare plots of the cross-correlation from which convection velocities could be computed for each set of measurements.

Later in the program several sets of data were processed on a HewlettPackard Correlator. In this case, the data reduction process was simpler because no separate analog-to-digital conversion was necessary. However, a separate operation was required to obtain spectral data. The results obtained from both techniques were substantially the same.

Figures 14 and 15 show cross-correlations measured in the jet core for $M=0.3$ with the upstream wire located at $X / D=2$ and $X / D=4$. Both broad band results and results for a one-octave-wide band centered about the spectral peak of $1300 \mathrm{~Hz}$ are shown. There is a peak in the cross-correlation at a wire spacing of $\frac{1}{2}$ inch (this peak is 0.96); for a wire spacing of $\frac{1}{8}$ inch, the peak occurs at a negative time delay. An effort is under way to understand this wave pattern. 


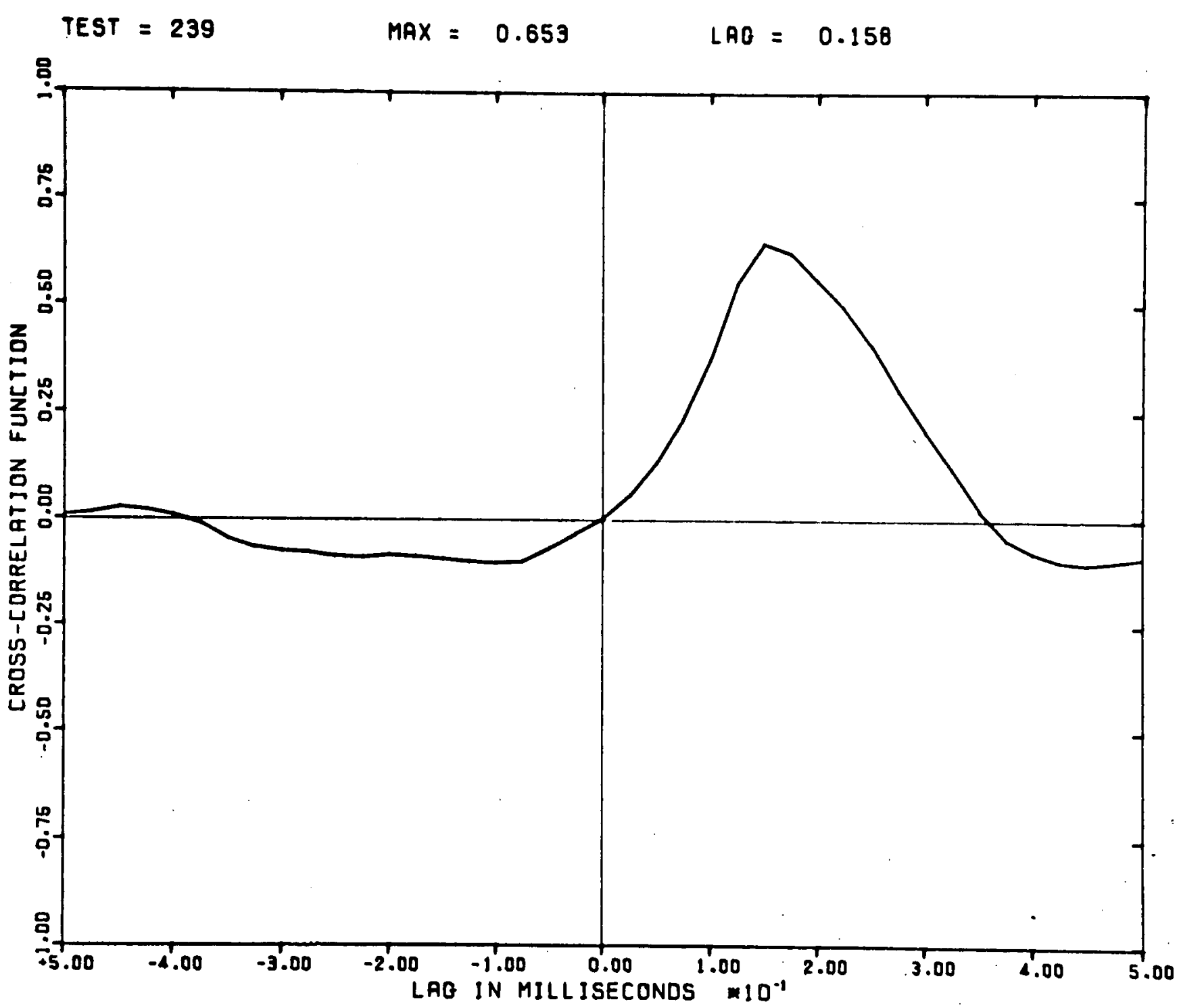

FI GURE 13 COMPUTER PRINTOUT OF TYPICAL CROSS-CORRELATION, $M=0.3, X / D=2, Y / D=0.4$ 

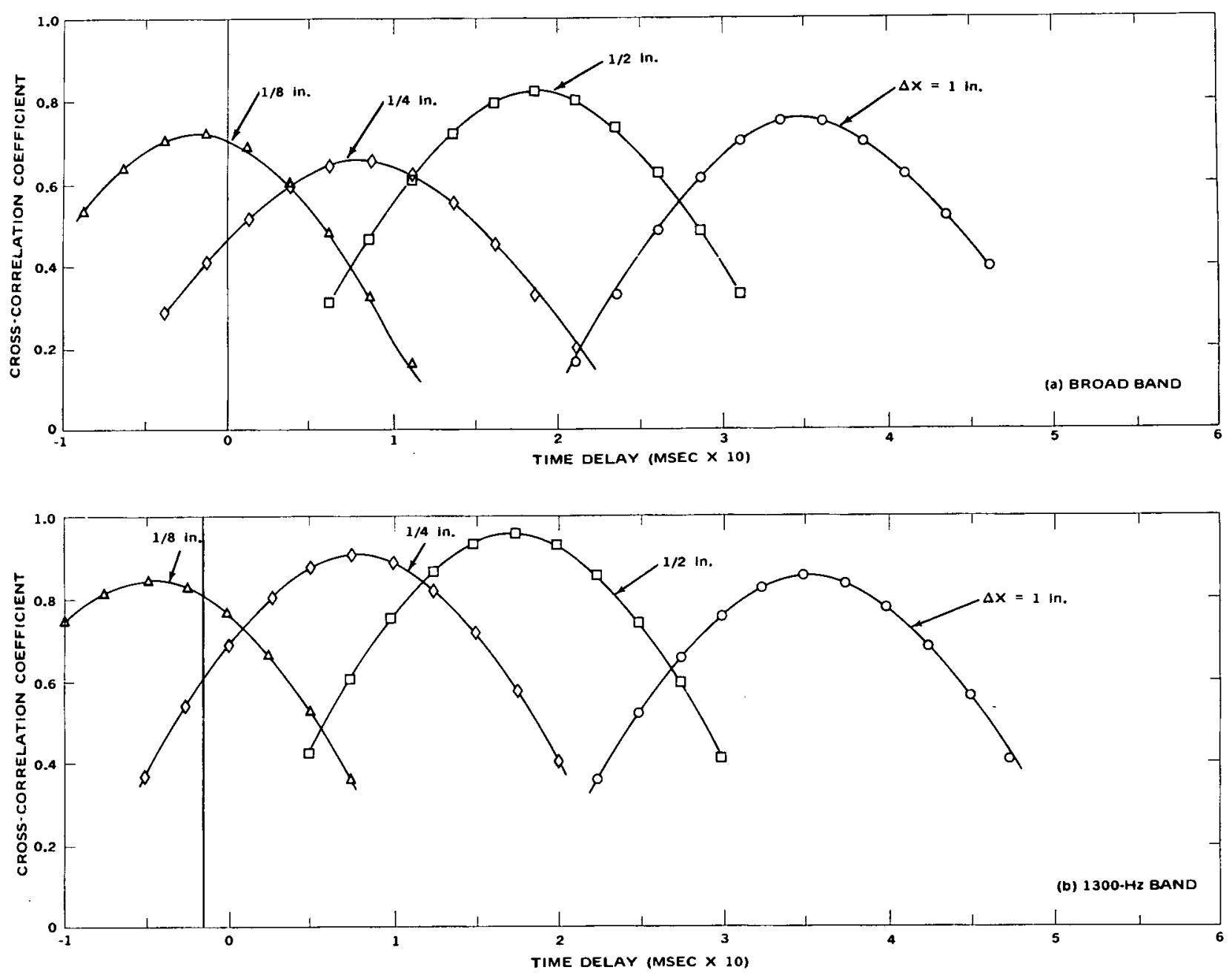

FIGURE 14 CROSS-CORRELATION OF HOT WIRE SIGNALS IN JET CORE VERSUS TIME DELAY FOR
SELECTED HOT WIRE SPACINGS, $M=0.3, Y / D=0.133$, UPSTREAM WIRE AT $X / D=2$ 

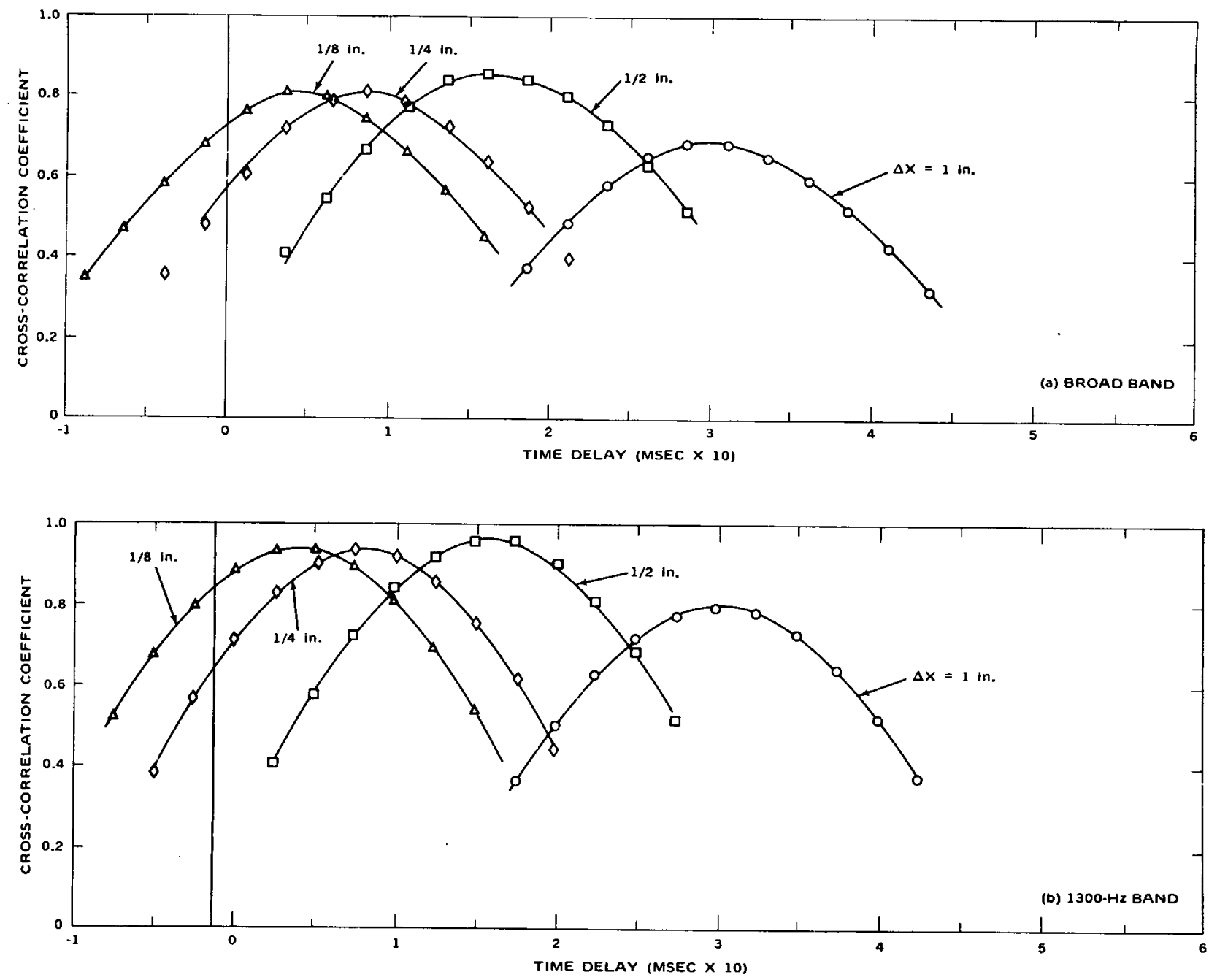

FIGURE 15 CROSS-CORRELATIONS OF HOT WIRE SIGNALS IN JET CORE VERSUS TIME DELAY FOR SELECTED HOT WIRE SPACINGS, $M=0.3, Y / D=0$, UPSTREAM WIRE AT $X / D=4$ 
Figures 16 and 17 show cross-correlations measured near the lip of the jet under the same conditions. Here results are presented for the broad band and for one-actave-wide bands centered about $800 \mathrm{~Hz}, 1300 \mathrm{~Hz}, 1600 \mathrm{~Hz}$, and $3200 \mathrm{~Hz}$. In the mixing region near the lip, the flow is dominated by the turbulence, with the usual decay of cross-correlation with increasing wire spacing.

To obtain the time delay associated with each value of wire spacing, the envelope tangent to each correlation curve and passing through $R_{11}=1$ at zero time delay was drawn. This envelope's point of tangency then determined the time delay to be used with each wire spacing to compute convection velocity. The time delay determined in this way does not correspond to the time delay for maximum cross-correlation but rather to the time delay associated with a moving frame in which the rate of decay of the correlation is a minimum. The envelope represents the autocorrelation in the moving frame. In cases where scatter in the measured results caused some curves to deviate from the envelope (see, for example, Figure 16d), the curves were shifted vertically to determine the time delay to be used with the given wire spacing. The resulting convection velocities are discussed in the next section. 

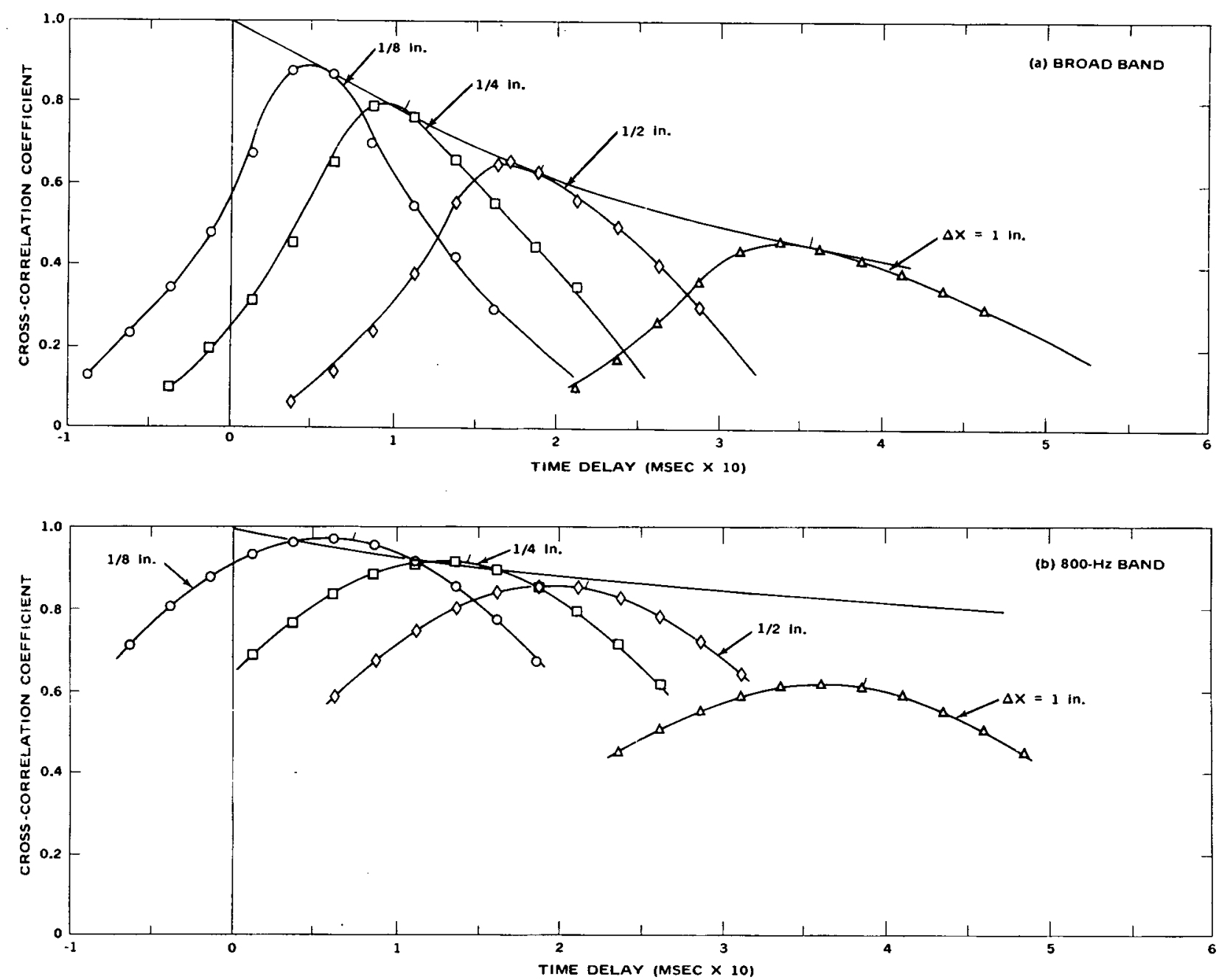
FIGURE 16 CROSS-CORRELATIONS OF AXIAL VELOCITY FLUCTUATION VERSUS TIME DELAY FOR
SELECTED HOT WIRE SPACINGS, $M=0.3, Y / D=0.4$, UPSTREAM WIRE AT $X / D=2$ 

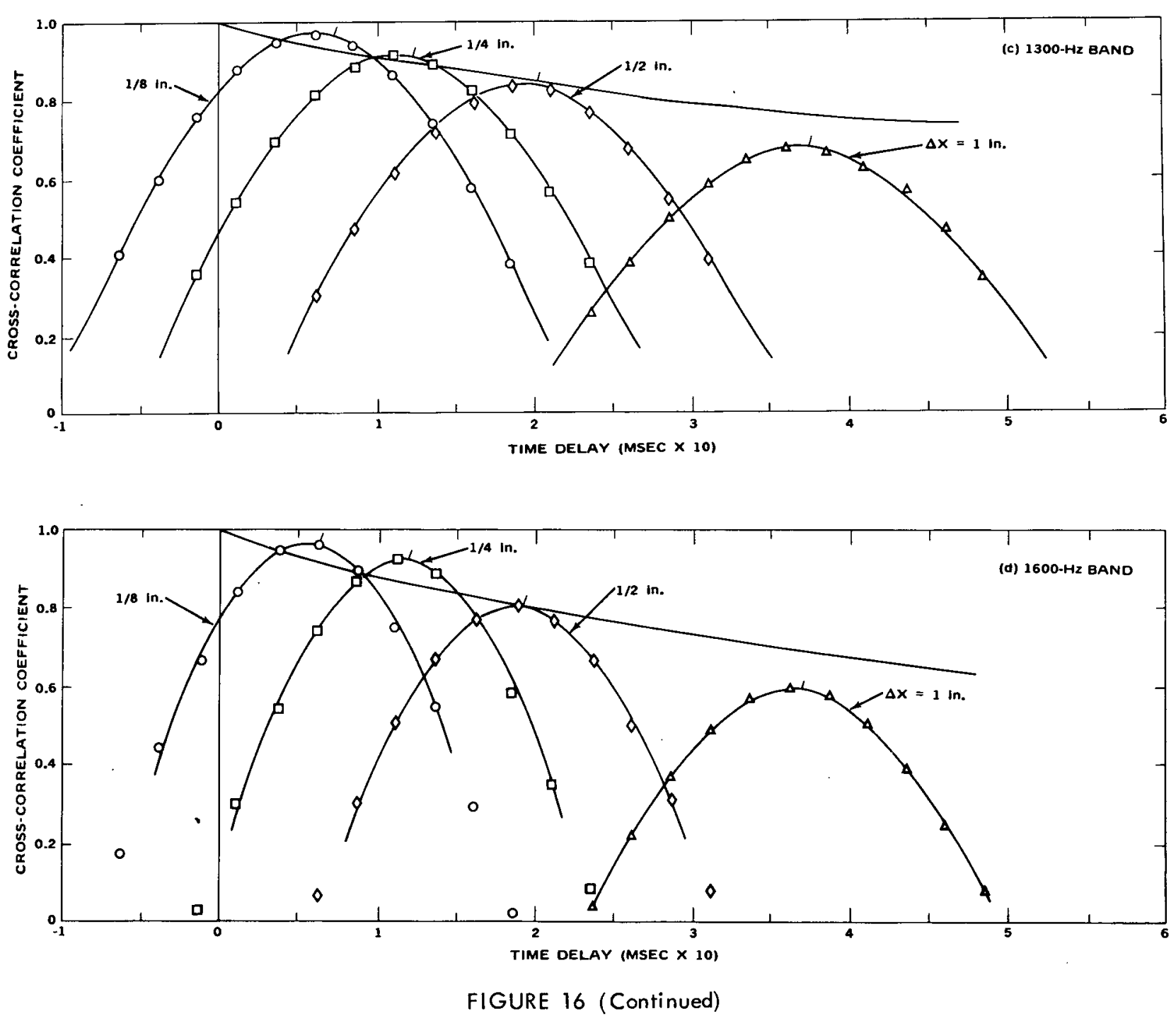


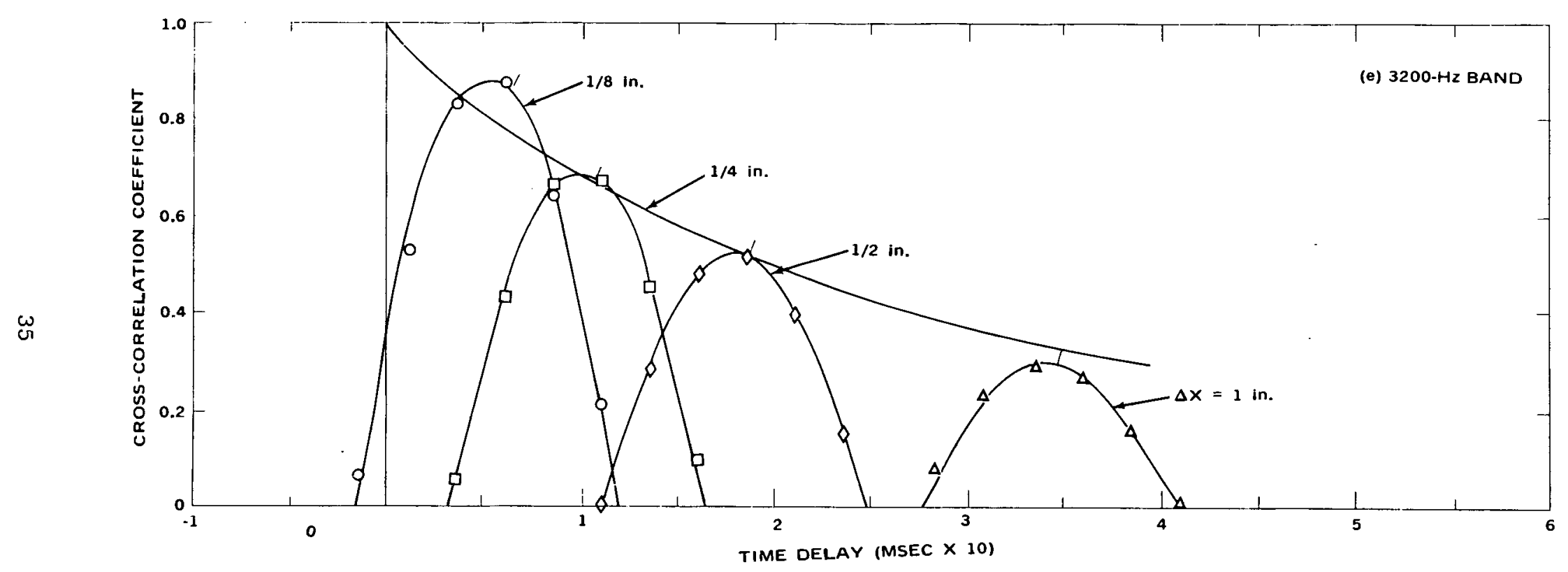

FIGURE 16 (Concluded) 

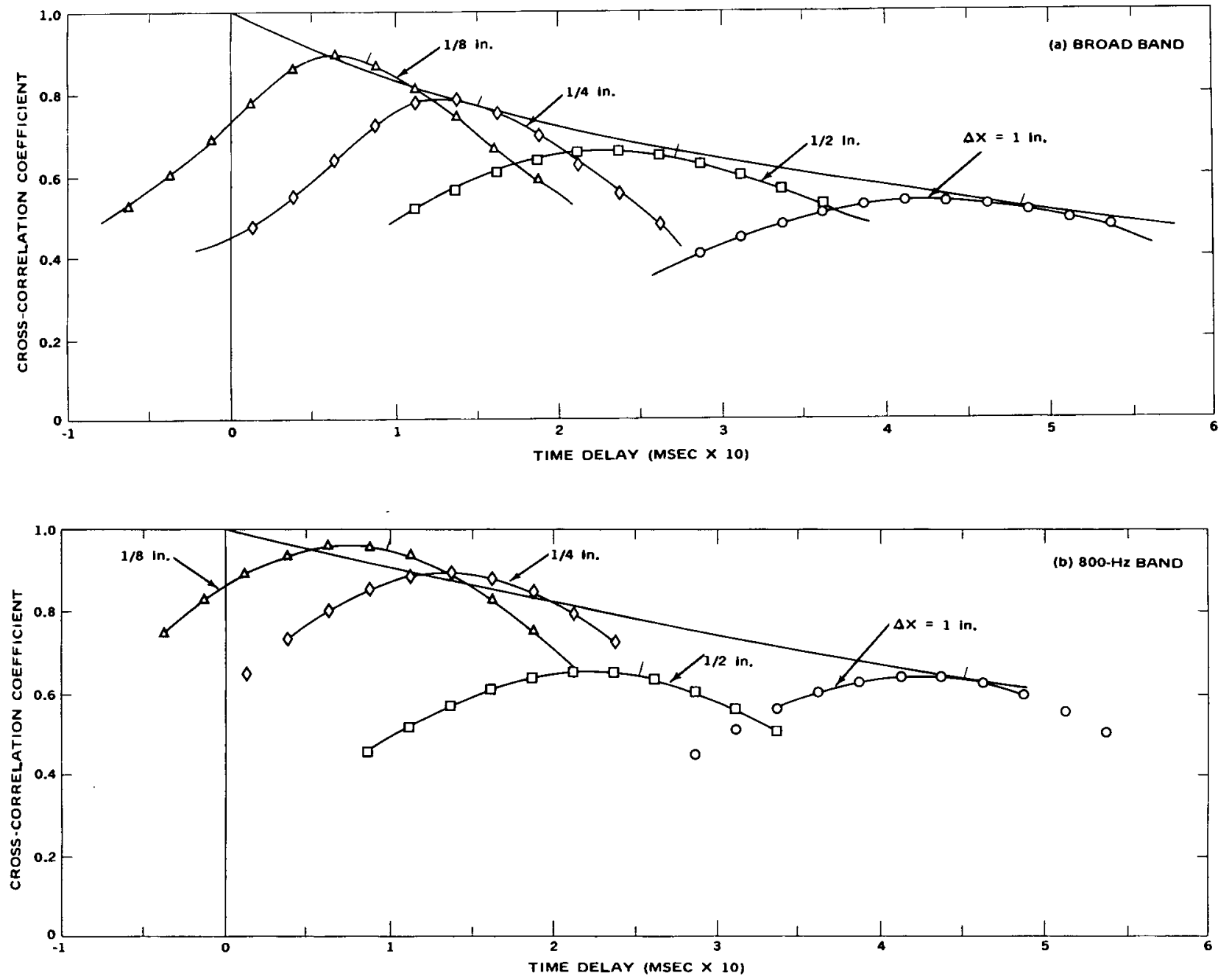

FIGURE 17 CROSS-CORRELATIONS OF AXIAL VELOCITY FLUCTUATION VERSUS TIME DELAY FOR SELECTED HOT WIRE SPACINGS, $M=0.3, Y / D=0.53$, UPSTREAM WIRE AT $X / D=4$ 

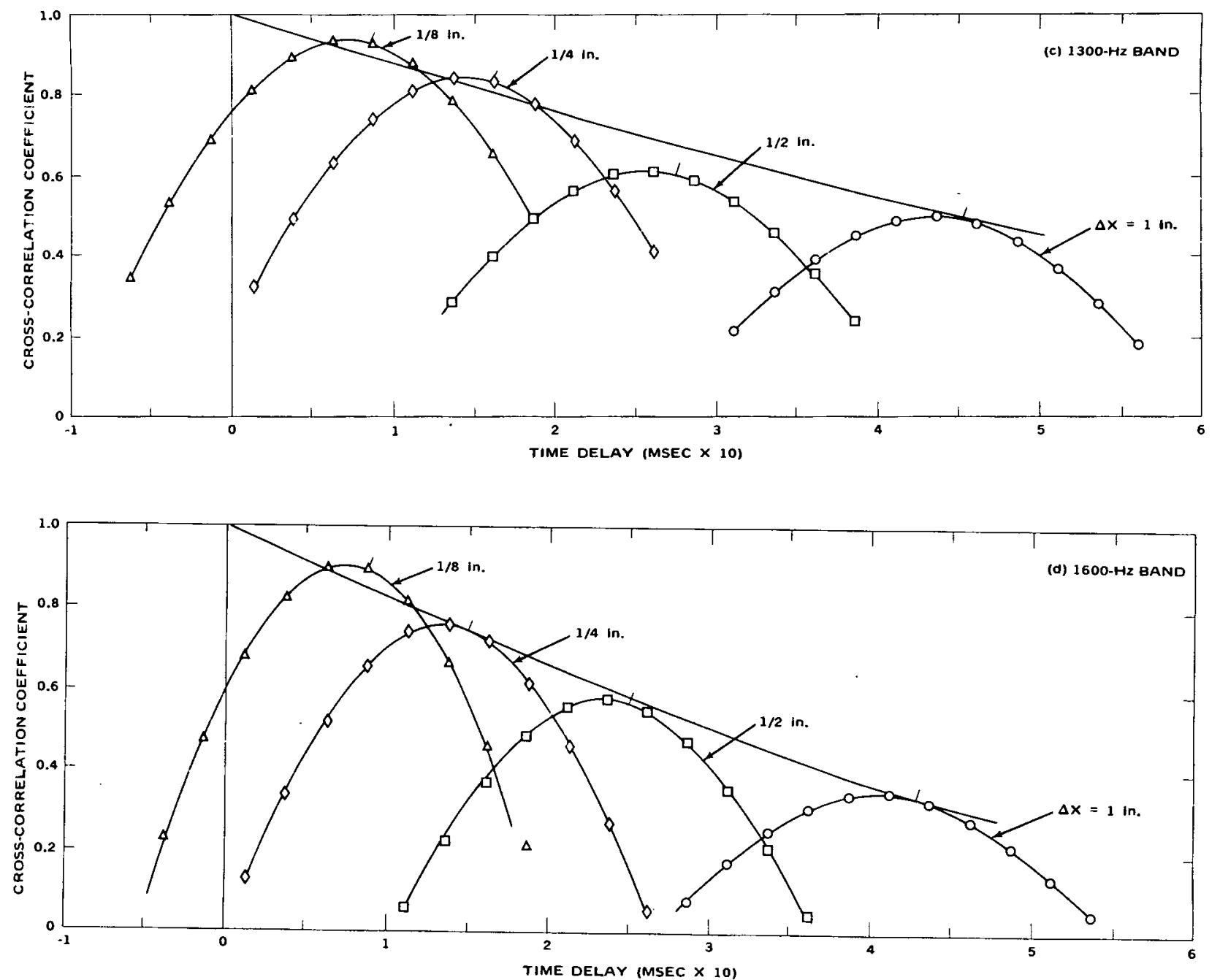

FIGURE 17 (Continued) 


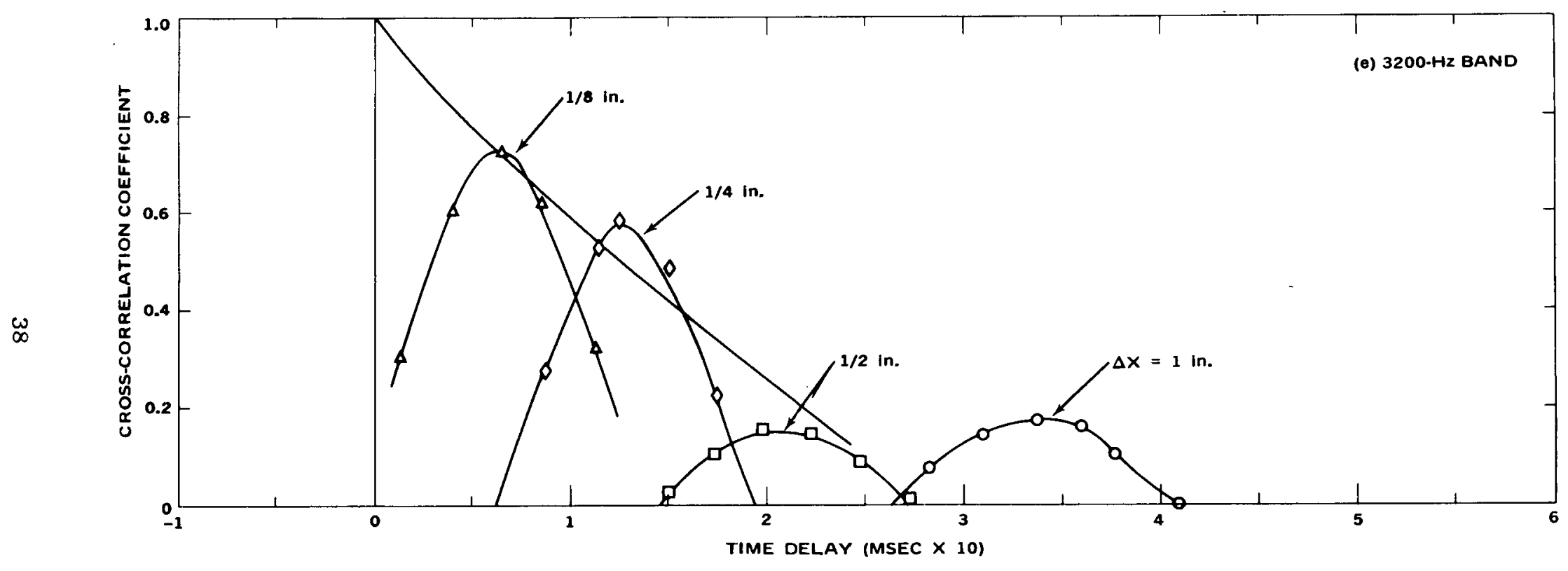

FIGURE 17 (Concluded) 


\section{CONVECTION VELOCITY MEASUREMENTS}

Convection velocities in jet turbulence have been measured by several investigators. Davies et al ${ }^{16,17}$ reported convection velocity measurements in which the axial convection velocity profile, scanned radially across the jet, was compared with the mean axial velocity profile. They found that, for the radial position $\mathrm{Y}<\mathrm{D} / 2$, the convection velocity $\mathrm{U}_{\mathrm{c}}$ was less than the mean axial velocity $U$ and, for $\mathrm{Y}>\mathrm{D} / 2, \mathrm{U}_{\mathrm{c}}$ was greater than $\mathrm{U}$. Bradshaw, Ferriss, and Johnson 18 reported similar results, as did Wills. ${ }^{22}$ That the axial convection velocity varies more slowly across the shear layer adjacent to the jet core than does the mean velocity is thus well established experimentally. Davies, Fisher, and Barratt ${ }^{16}$ and Fisher and Davies ${ }^{23}$ explain this phenomenon physically in terms of the probability density of the turbulent velocity fluctuations. Basically, the large scale fluctuations to one side of the center line of the shear layer contain large components of the characteristic free stream velocity just outside the shear layer. That is, on the jet core side $(Y<D / 2)$ of the center of the shear layer, the large scale velocity fluctuations carry significant energy characteristic of the core velocity. Fisher and Davies ${ }^{23}$ explain how these eddies influence the cross-correlation measurements so as to cause the observed differences between the mean velocity and convection velocity across the mixing region.

In addition to the overall convection velocity measurements, Fisher and Davies $^{23}$ measured convection velocity for several frequency bands. Their measurements were made to determine whether or not various wave-number (frequency) components are convected downstream at the same velocity. Their data were taken only for the radial position $\mathrm{Y} / \mathrm{D}=\frac{1}{2}$ and indicated that the convection velocity increased slightly with increasing center-band frequency 
of the filtered signals. Until the present work, it was still not clear, however, how the various frequency components are convected at different radial positions in the jet. In the following, measurements of axial convection velocity profiles across the jet for broad band (unfiltered), and for selected, numerically filtered narrow bands are reported. These results are based on the cross-correlation measurements described in the previous section.

The convection velocity that is computed from the cross-correlation measurements shows a consistent increase in value with wire spacing. This effect was discussed by Willmarth and Wooldridge ${ }^{24}$ for measurements in a boundary layer which exhibits similar behavior. Convection velocities for the broad band and for the various narrow frequency bands are plotted as a function of wire spacing in Figure 18 for locations near the center of the shear layer at two axial distances. (Figure 18a corresponds to data taken from the cross-correlation plots shown in Figure 16, and Figure 18b corresponds to those taken from Figure 17). The decrease in convection velocity with wire spacing was not observed by other investigators because their minimum wire spacing was too large; for wire spacings $\frac{1}{2}$ inch and greater, the convection velocity asymptotically approaches a constant value (Figure 18); thus, there are two possible interpretations of convection velocity measured in this way: (l) that corresponding to zero wire spacing, which is given by the zero intercept of the $U_{c}$ versus wire spacing curves; and (2) the asymptotic value of $U_{C}$ for large wire spacing.

The distribution of convection velocity across the jet for the broad band and for four narrow frequency bands is shown in Figure 19 for $\mathrm{X} / \mathrm{D}=2$ and $M=0.3$. The asymptotic values at large spacing agree with the data obtained by Davies et al. ${ }^{16}$ in the outer portion of the mixing layer but are somewhat higher in the inner portion. The intercept values, Figure 18b, are much lower, joining the mean velocity curve near the outer edge of the mixing region. The narrow frequency band data obtained by Davies et al. 16 

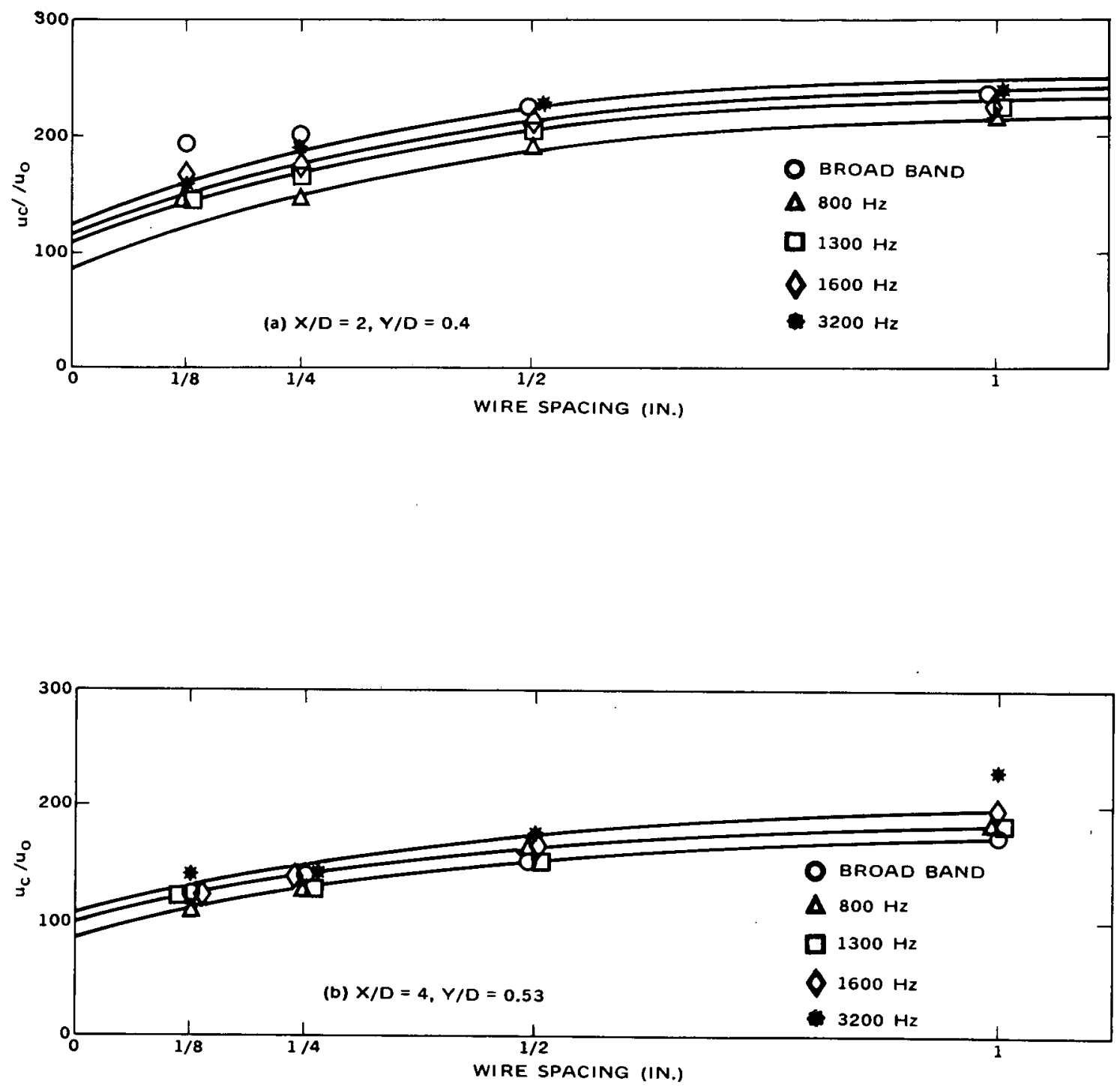

FIGURE 18 CONVECTION VELOCITY VERSUS WIRE SPACING 


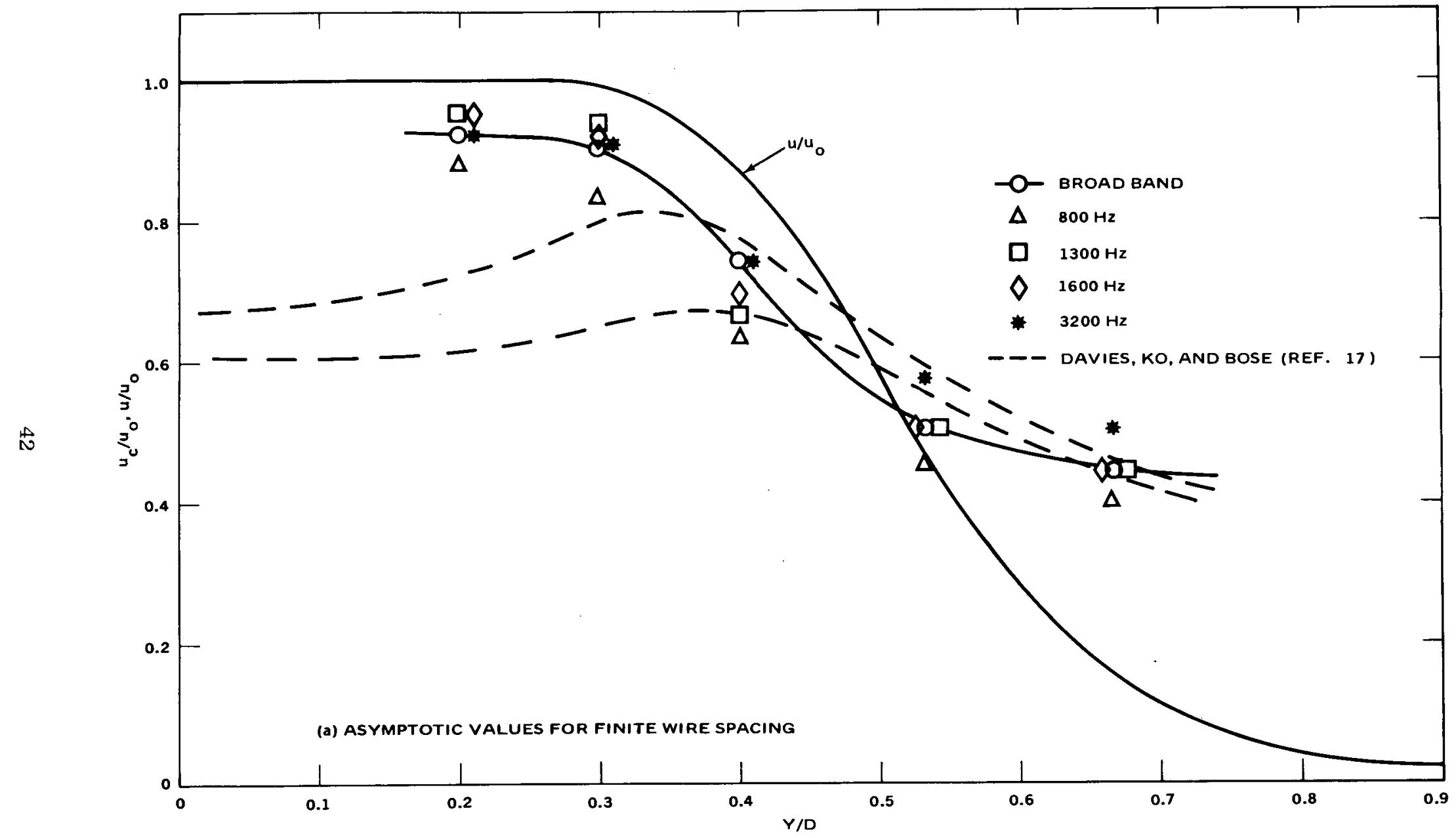

FIGURE 19 CONVECTION VELOCITY PROFILES ACROSS THE JET, $M=0.3, X / D=2$ 


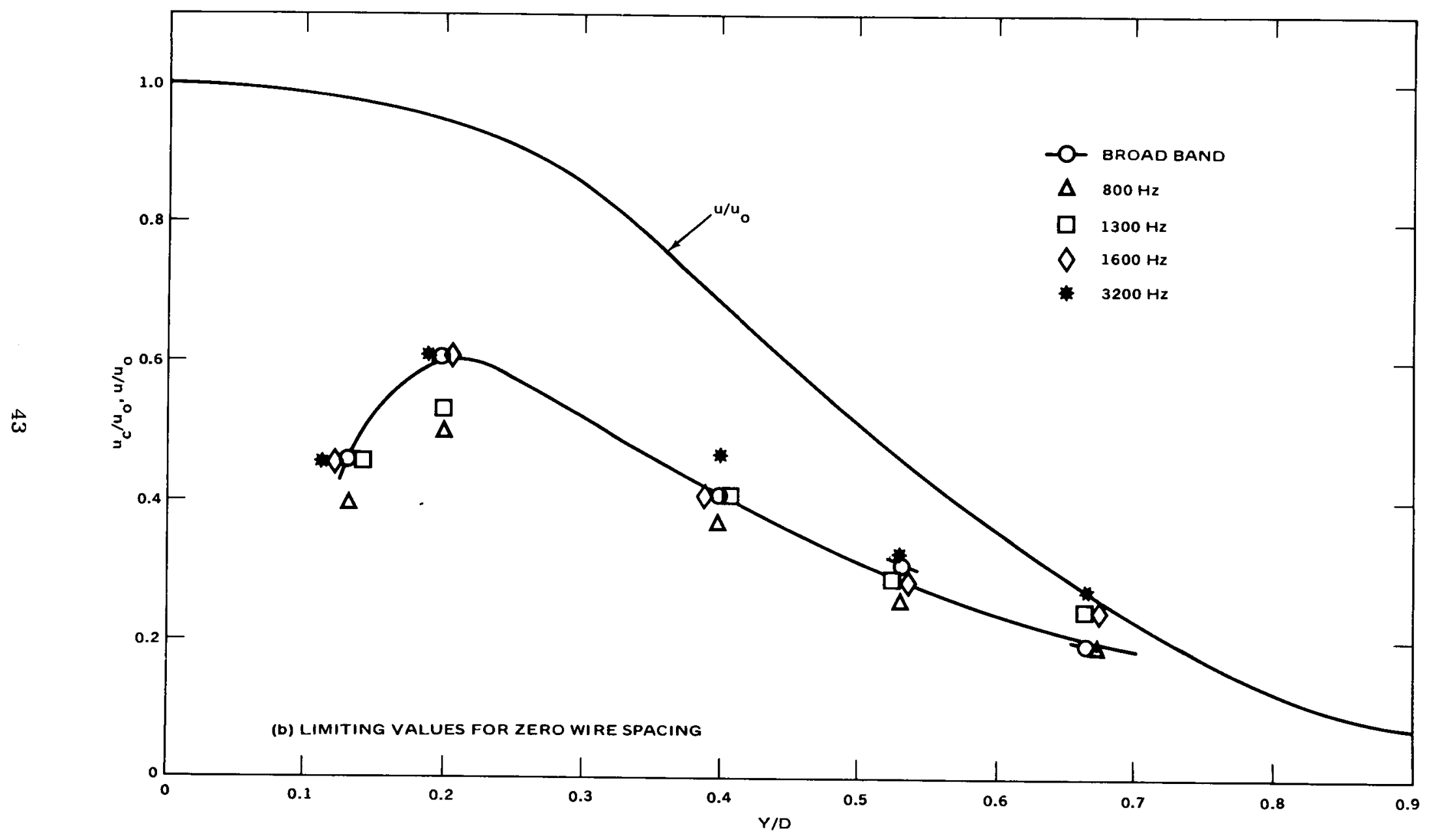

FIGURE 19 (Concluded) 
at $\mathrm{Y} / \mathrm{D}=0.5$ indicated that the higher frequency components are convected faster than the lower frequency components. The current data show that this behavior holds true across the entire mixing region.

Similar convection velocity profiles for $\mathrm{X} / \mathrm{D}=4$ are shown in Figure 20 . The asymptotic values for large wire spacing follow more closely the mean velocity. However, the limiting values of convection velocity for zero wire spacing are also somewhat lower and do not join the mean velocity curve until they reach the outer portion of the mixing region. In addition to the $M=0.3$ data, convection velocities at $M=0.5$, measured during the preceding year's work for three radial positions, are shown in Figure 21. The general result-that lower frequency components travel slower--is also evident in the $M=0.5$ data.

Clearly there is a large difference between the value of convection velocity at zero wire spacing and the asymptotic value for large wire spacing. One must use great care, therefore, in attempting to interpret the meaning of such measurements. Additional thought needs to be given not only to the definition of a convection velocity but, more importantly, also to an understanding of its meaning in terms of the production of jet noise.

The meaning of the convection velocity can be illustrated by Figure 22 , which shows space-time correlation contours. The definition of convection velocity defined by the method outlined above is based on the time delay corresponding to the vertical tangent to any contour. With this approach, the convection velocity noticeably decreases for small times, because of the finite width of the contours. This is equivalent to saying with respect to Figure 16 that the difference in time delay between the peak point and the tangency point on each curve becomes small compared to the total time delay at large wire spacing. 


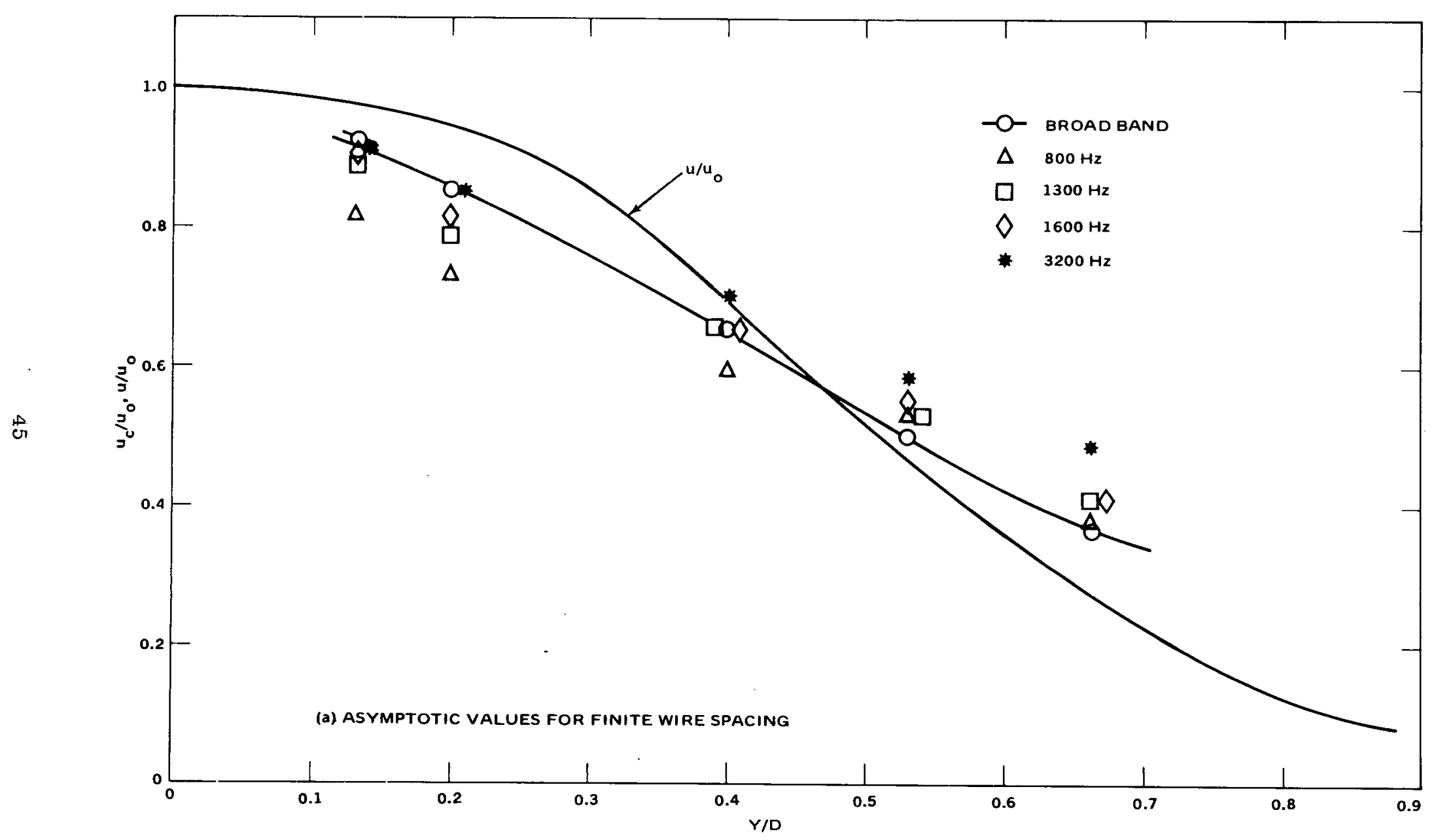

FIGURE 20 CONVECTION VELOCITY PROFILES ACROSS THE JET, $M=0.3, X / D=4$ 


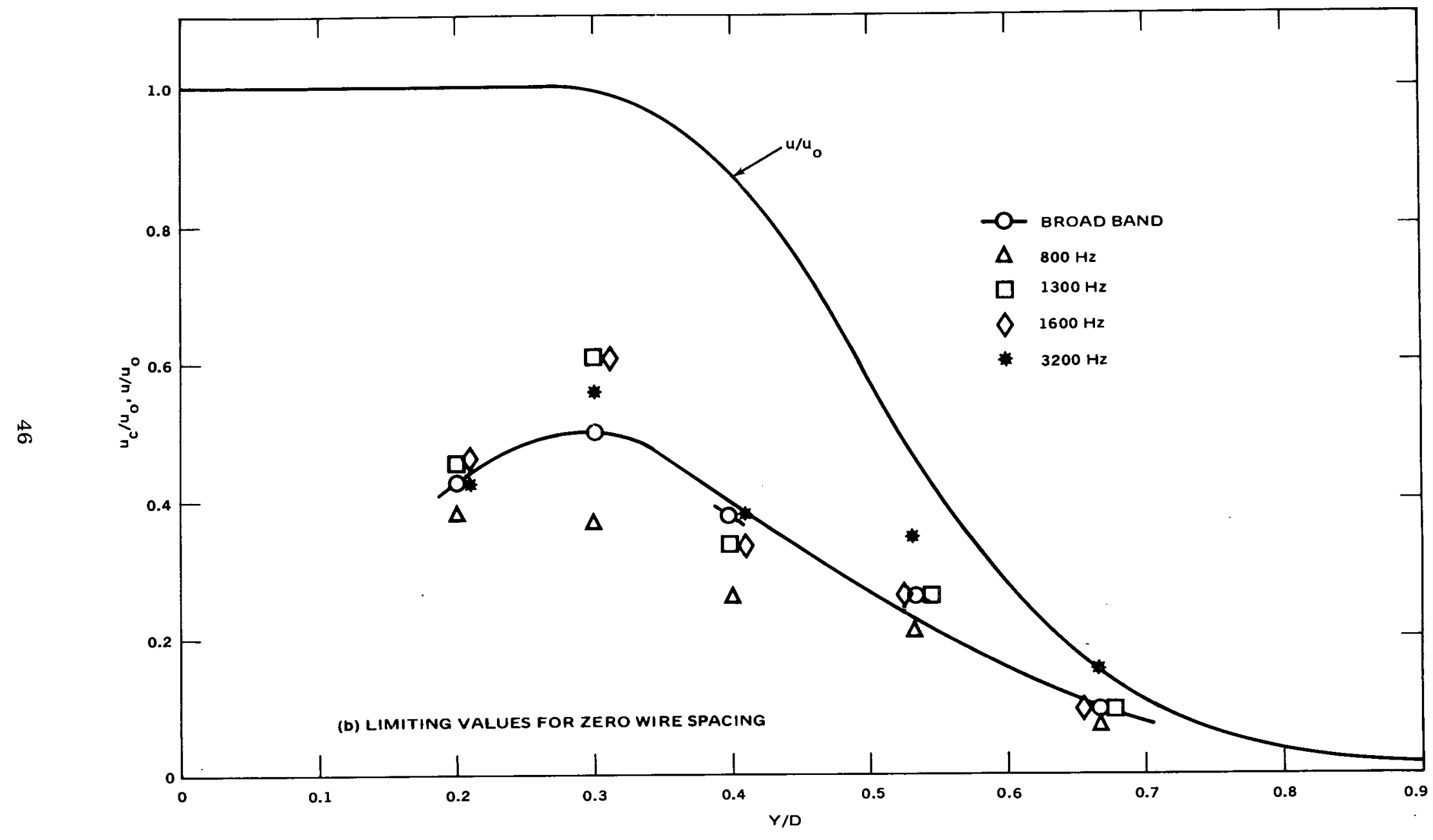

FIGURE 20 (Concluded) 


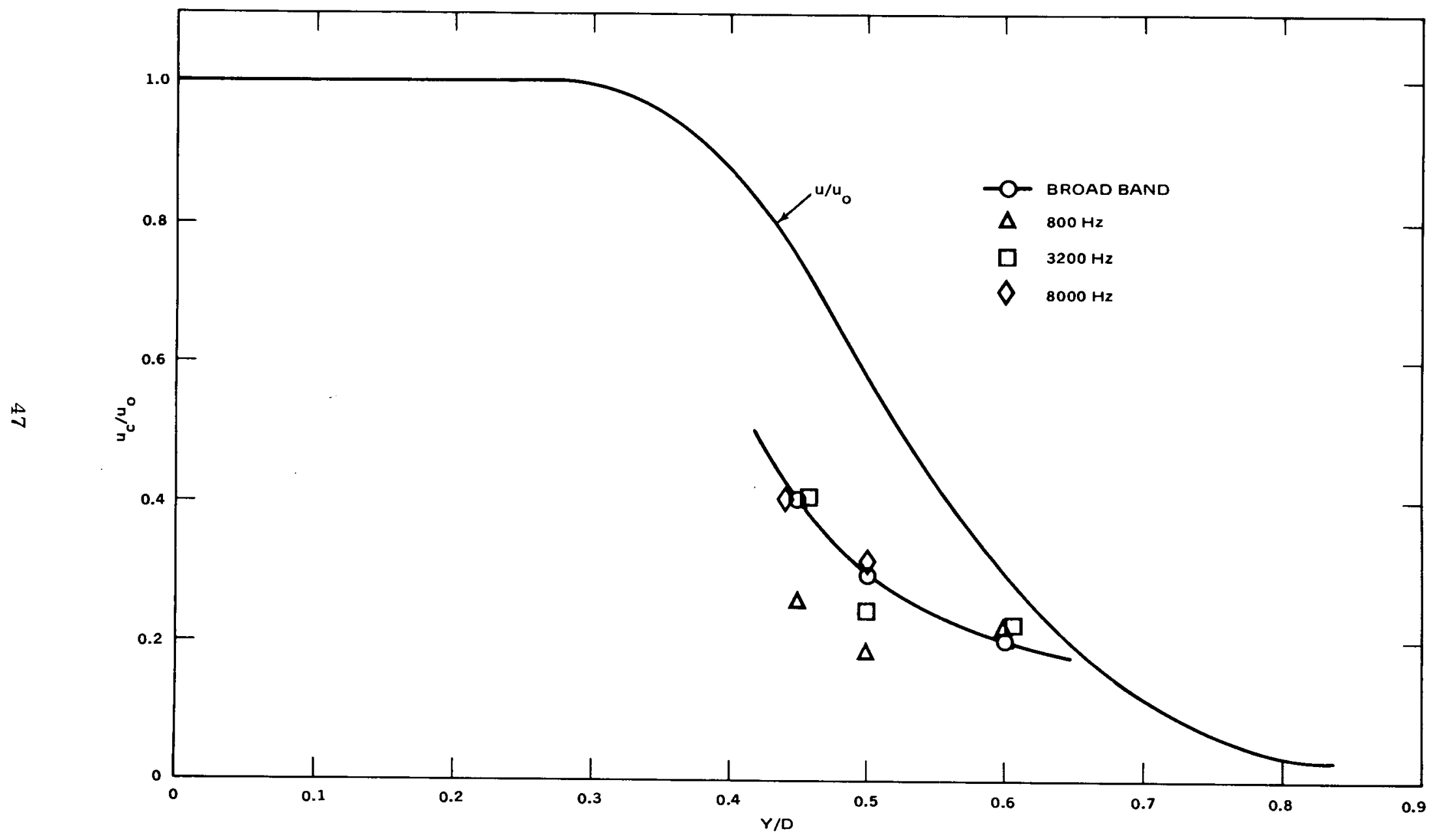

FIGURE 21 CONVECTION VELOCITY PROFILES ACROSS THE JET, ASYMPTOTIC VALUES FOR LARGE WIRE SPACING, $M=0.5, X / D=2$ 

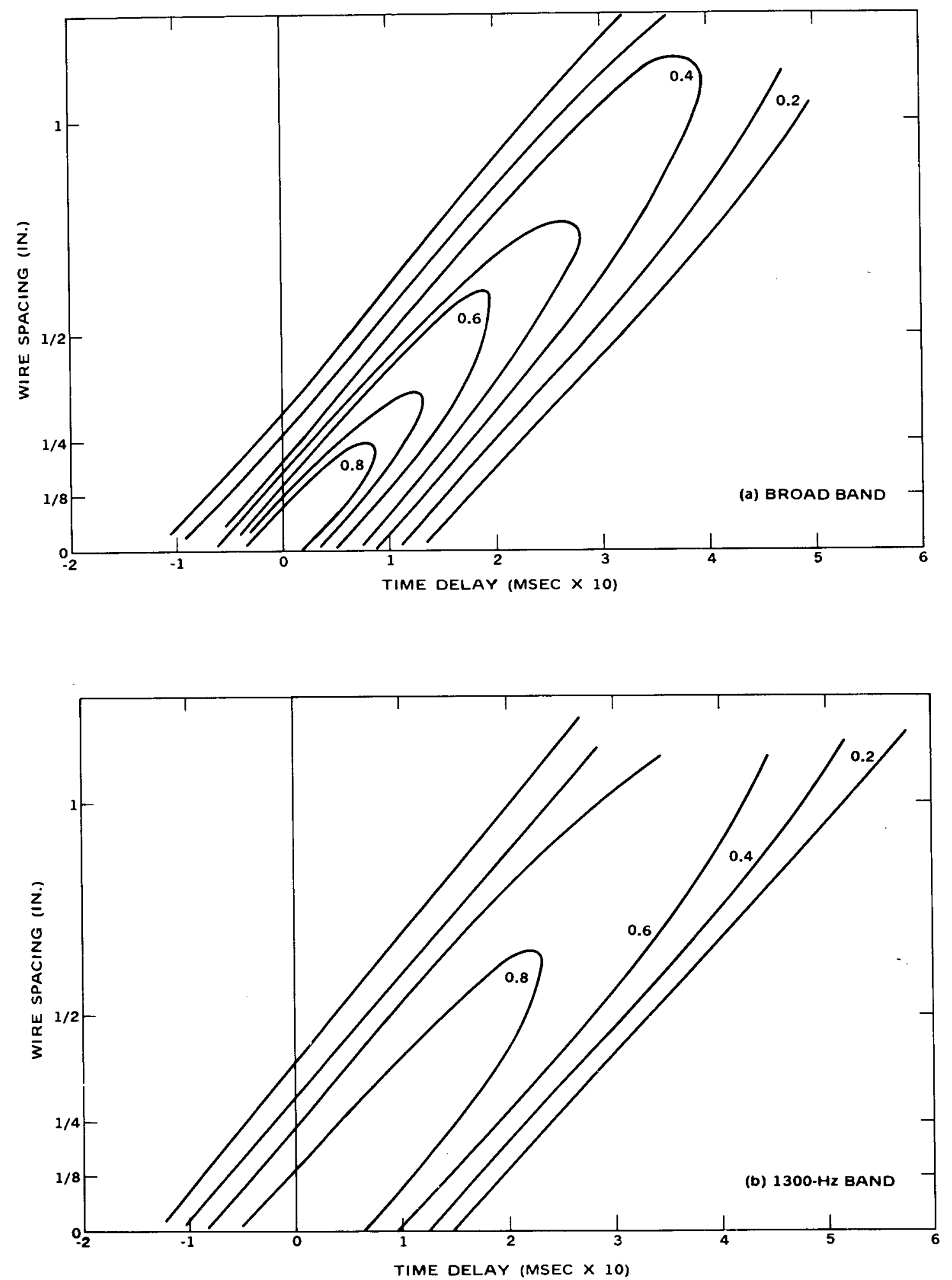

FIGURE 22 SPACE-TIME CORRELATION CONTOURS, $M=0.3, X / D=2, Y / D=0.4$ 


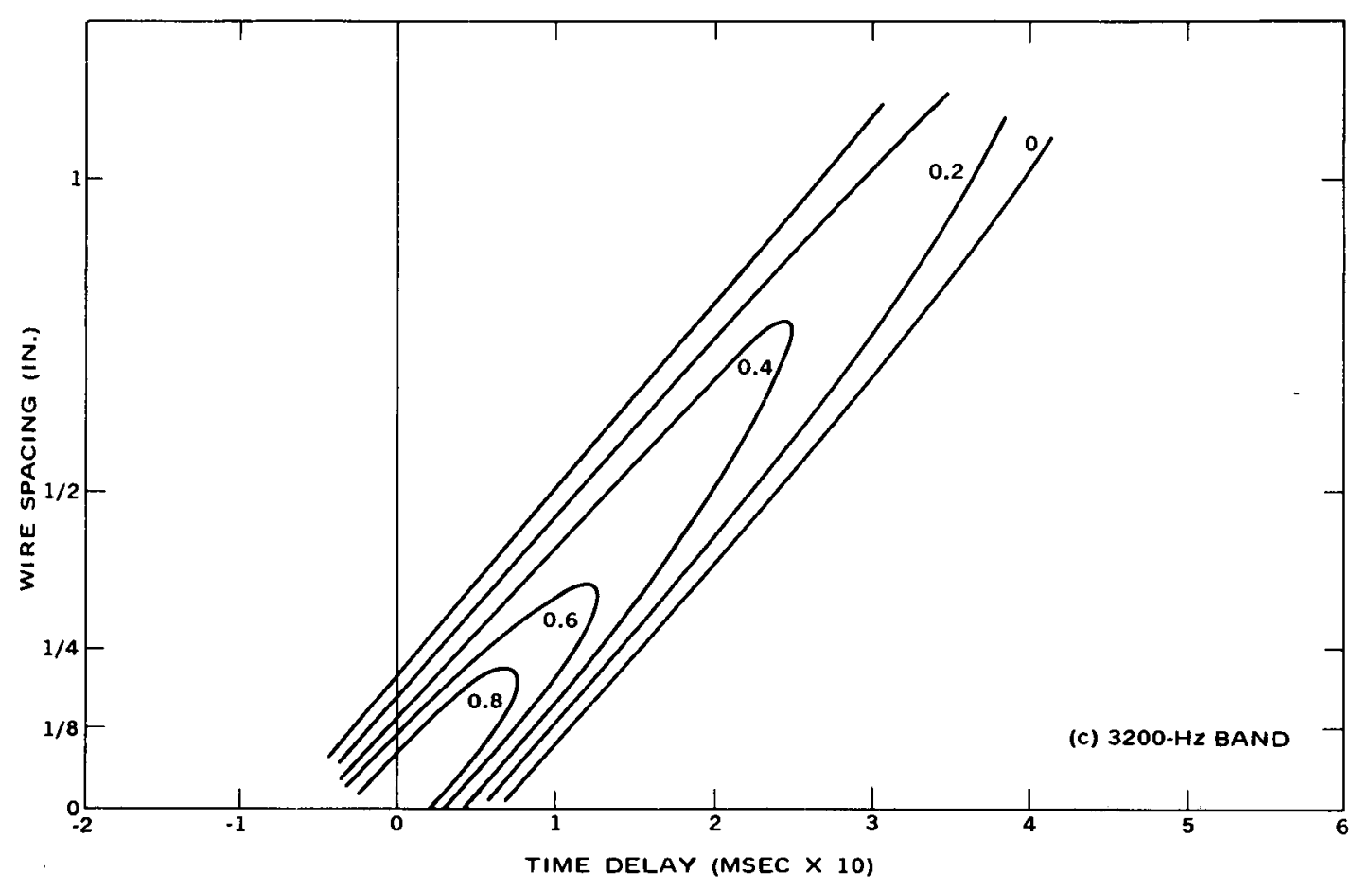

FIGURE 22 (Concluded) 


\section{THE EFFECT OF EVAPORATING DROPLETS ON SOUND GENERATION BY JET TURBULENCE}

Sound generation by turbulent, two-phase flow was considered recently by Crighton and Ffowcs Williams, ${ }^{25}$ who used the methods of Lighthill's theory of aerodynamic noise to consider sound generation for the case of a distribution of small gas bubbles in a liquid and for the case of a dusty gas. They found that monopole radiation due to the volumetric response of the bubbles to the turbulent pressure field can be an order of magnitude greater than the quadrupole equivalent of the turbulent flow alone. In the case of a dusty gas, which is of more interest in aerodynamic jet noise work, only dipole and quadrupole sources are present; Crighton and Ffowcs Williams, 25 in fact, showed that the dipole radiation is equivalent to an increase in the usual quadrupole radiation. This increase depends on the mass concentration of dust and is significant only for mass concentrations too large (in excess of unity) to be of practical importance in aircraft jet noise applications.

The important case of sound generation by a turbulent, two-phase flow that has not yet been considered is that of a condensible aerosol. Condensation and evaporation from liquid droplets in a turbulent flow field provide a mechanism for the production of monopole radiation. In this section, the monopole acoustic source strength radiated from a finite turbulent flow region containing condensible droplets is considered. The droplets exchange mass, momentum, and energy with the gaseous phase, which is taken to be a mixture of inert gas and vapor. The manner in which these exchange processes enter into the equations describing the gas and droplet flow has been discussed in detail by Marble 26 and Marble and Wooten. ${ }^{27}$ For example, the vapor production rate appears as a mass-source term in the continuity 
equation for the gas phase and as a mass-sink term in the corresponding equation for the droplet cloud. A nonhomogeneous wave equation is derived below in which there appears a monopole distribution arising from the mass exchange between phases. The monopole term is similar to the term studied by Crighton and Ffowcs Williams, ${ }^{25}$ which was due to forced oscillation of air bubbles in water. The monopole power output in the present case is not significant for water droplets in air at ambient temperatures $\left(\sim 70^{\circ} \mathrm{F}\right)$ but increases exponentially with temperature; at temperatures nearer boiling, the monopole radiation may be much greater than that due to the quadrupoles equivalent to the turbulent flow alone.

In the following analysis, the droplet cloud is treated as a continuumlike flow in the usual manner. The gas phase is taken as a mixture of inert gas and vapor. Since the liquid density is much greater than that of the gas, the volume fraction of liquid is neglected in the conservation equations for the gas phase. In addition, the continuum assumption applied to the droplet cloud is considered to be applicable in treating the turbulent flow dynamics and the acoustic field; that is, the droplet diameter and average droplet spacing are assumed to be much smaller than the microscale of the turbulent fluctuations and the wavelength of the acoustic waves. A related assumption also taken here is that locally the droplets remain in mechanical and thermodynamic equilibrium with the gas flow. These assumptions are similar to those made by Crighton and Ffowcs Williams, ${ }^{25}$ who discussed their range of validity in some detail.

To formulate a Lighthill equation for the gaseous phase, we begin with the conservation of mass for this phase expressed by

$$
\frac{\partial}{\partial t} \rho+\frac{\partial}{\rho x_{i}}\left(\rho u_{i}\right)=Q
$$


where $\rho$ and $u_{i}$ are the density and velocity of the gas phase and $Q$ is the rate of mass addition to the gas phase per unit volume by evaporation from the droplet cloud. The momentum equation for the gas phase may be written as

$$
\frac{\partial}{\partial t}\left(\rho u_{i}\right)+\frac{\partial}{\partial x_{j}}\left(\rho u_{i} u_{j}\right)+\frac{\partial}{\partial x_{j}} P_{i j}=F_{i}
$$

where $P_{i j}$ is the stress tensor and $F_{i}$ is force per unit volume exerted by the droplet cloud on the gas phase. Combination of Eqs 1 and 2 in the usual manner gives

$$
\frac{\partial^{2}}{\partial t^{2}} \rho-C_{o}^{2} \nabla^{2} \rho=\frac{\partial Q}{\partial t}-\frac{\partial F_{i}}{\partial x_{i}}+\frac{\partial^{2} T_{i j}}{\partial x_{i} \partial x_{j}}
$$

where

$$
\mathrm{T}_{i j}=\rho u_{i} u_{j}+P_{i j}-C_{o}^{2} \rho \delta_{i j}
$$

The sound speed $\mathrm{C}_{\mathrm{O}}$ in these equations is the speed of sound in the ambient region external to the jet. For this reason, the fluctuations in $p$ do not cancel the $\mathrm{C}_{\mathrm{O}}^{2}$ term, as in the Lighthill formulation, for single phase turbulent flow. ${ }^{2}$ On the other hand, if the assumption of equilibrium between phases is strictly true, fluctuations in p are approximately equal to fluctuations in $\mathrm{C}_{\mathrm{e}}^{2}\left(\rho+\rho_{\mathrm{p}}\right)$ where $\mathrm{C}_{\mathrm{e}}$ is the equilibrium sound speed in the gas-droplet mixture and $\rho_{p}$ is the density of droplets. The equilibrium sound speed in a gas containing droplets is

$$
\mathrm{C}_{\mathrm{e}}^{2}=\mathrm{C}^{2}\left\{\gamma\left(1+\mathrm{K}_{\mathrm{p}}\right)\left[1-2\left(\frac{\gamma-1}{\gamma}\right) \frac{1}{\eta}+\left(\frac{\gamma-1}{\gamma}\right) \frac{\left(1+\mathrm{K}_{\mathrm{p}} \mathrm{C}_{\ell} \mathrm{C}_{\mathrm{p}}\right)}{\eta^{2}}\right]\right\}^{-1}
$$


where $\mathrm{C}$ is the sound speed in the gas phase alone, $\gamma$ is the ratio of specific heats and $C_{p}$ is the specific heat at constant pressure of the gas, $\mathrm{C}_{\ell}$ is the specific heat of the liquid, $\mathrm{K}_{\mathrm{p}}$ is the mass fraction of liquid, and $\eta$ is the specific heat parameter given by

$$
\eta=\stackrel{h}{l} / \mathrm{c}_{\mathrm{p}} \mathrm{T}
$$

where $h_{\ell}$ is the latent heat of vaporization of the liquid. In general $\mathrm{C}_{e}$ is much less than $\mathrm{C}$ (which is approximately equal to $\mathrm{C}_{0}$ ). Also, in many cases of practical significance, nonequilibrium between the liquid and gas phases is important. Therefore, the usual ordering of terms in Eq 3 is not so straightforward as in the case of a single phase fluid. We will defer consideration of the effects due to different sound speeds and nonequilibrium until later and consider here only the monopole source term.

The density disturbance in the far field, obtained from the usual retarded potential solution is

$$
\left(\rho-\rho_{0}\right)(\vec{x}, t)=\frac{1}{4 \pi c_{0}^{2}} \int_{\vec{y}} \frac{\partial Q}{\partial t}\left(\vec{y}, t-\frac{1 \vec{x}-\vec{y}^{1}}{C_{0}}\right) \frac{d \vec{y}}{1 \vec{x}-\vec{y} 1}
$$

It follows that the monopole acoustic power output $P_{m}$ in from the region containing droplets, whose volume is of order $L^{3}$ is

$$
P_{m} \sim \frac{1}{4 \pi C_{o}^{2}}\left\langle\left(\frac{\partial Q}{\partial t}\right)^{2}\right\rangle l_{o}^{3} L^{3}
$$

where $\rho_{0}$ is the density of the gas in the far field and the brackets $\langle>$ denote time-average values. Expression 8 is obtained from Eq 7 subject to the condition that differences in retarded time corresponding to points separated by an eddy scale $b_{0}$ are negligible compared with the time-scale 
of the source field $\ell_{0} / U_{O}$, where $U_{O}$ is the rms turbulent velocity. This requirement is discussed in detail by Lighthill. 2,3

Now we wish to express Eq 8 in terms of the physical parameters describing the gas-droplet, two-phase system. In the case of a droplet cloud in a gas phase consisting of air, inert gas, and vapor, the mass transfer rate (evaporation rate) is diffusion controlled. 24,25 The vapor fraction $\mathrm{K}_{\mathrm{V}}$ in the gas phase can be written

$$
\mathrm{K}_{\mathrm{v}}=\frac{\mathrm{P}}{\mathrm{P}}=\frac{1}{\beta} \frac{\rho}{\rho}
$$

where $\beta=\mathrm{m}_{\mathrm{v}} / \mathrm{m}$ is the ratio of the molecular weights of the inert gas and the vapor and $P_{V}$ is the equilibrium vapor pressure. In Eq 9 the gas phase is assumed to obey the ideal gas equation of state. In addition to the conservation of mass for the gas phase, a conservation of mass equation for the vapor phase may be written

$$
\frac{\partial}{\partial \mathbf{t}} \rho_{v}+\frac{\partial}{\partial \mathbf{x}_{\mathbf{i}}}\left(\rho_{\mathrm{v}} \mathrm{u}_{\mathbf{i}}\right)=\mathbf{Q}
$$

where $\rho_{\mathrm{V}}$ is the density of vapor. Combination of Eqs 1 and 10 give

$$
\beta K_{v}\left(\frac{\partial}{\partial t} \rho+\frac{\partial}{\partial x_{i}}\left(\rho u_{i}\right)\right)+\beta \rho\left(\frac{\partial}{\partial t} K_{v}+u_{i} \frac{\partial K}{\partial x_{i}}\right)=Q
$$

or

$$
\beta K_{V} Q+\beta \rho\left(\frac{D K_{V}}{D t}\right)=Q
$$

Therefore

$$
Q=\frac{\beta \rho}{1-\beta K_{v}} \frac{D_{V}}{D t}
$$


The equilibrium vapor fraction can be expressed using the clasius Clapeyron equation as follows

$$
\frac{\mathrm{K}_{\mathrm{v}}}{\mathrm{K}_{\mathrm{vo}}}=\frac{\mathrm{P}_{\mathrm{o}}}{\mathrm{P}} \exp \left[-\frac{\mathrm{h}_{\ell}}{\mathrm{RT}_{\mathrm{o}}}\left(\frac{\mathrm{T}_{\mathrm{o}}}{\mathrm{T}}-1\right)\right]
$$

where $\mathrm{K}_{\mathrm{vo}}$ and $\mathrm{P}_{\mathrm{O}}$ are vapor fraction and pressure at ambient conditions, and $\mathrm{K}_{\mathrm{V}}$ and $\mathrm{P}$ represent fluctuations about the ambient values. The same notation applies to temperature $\mathrm{T}$. Note that the equilibrium assumption implies that the liquid and gas phases are locally at the same temperature. Thus, $\mathrm{K}_{\mathrm{V}}$ is the equilibrium vapor pressure at $\mathrm{T}$. From Eqs 13 and 14 , then

$$
Q=\frac{\beta K v}{1-\beta K_{v}}\left[\eta\left(\frac{\gamma}{\gamma-1}\right)^{2}-1\right]\left(\frac{\rho}{P}\right) \frac{D p}{D t}
$$

where it is assumed that the pressure and temperature fluctuations are related by

$$
\frac{\mathrm{dP}}{\mathbf{P}}=\frac{\gamma-1}{\gamma} \frac{\mathrm{dT}}{\mathrm{T}}
$$

The fluctuation $P$ is taken as the fluctuation in the eddy motion pressure and estimated as the typical fluctuation in $\frac{1}{2} \rho_{\mathrm{O}} \mathrm{U}^{2}$ as was done by Crighton and Ffowcs Williams. ${ }^{25}$ Thus $P \sim \rho_{0} \sigma U^{2}$, where $\sigma=u_{O} / U$ is the relative turbulence level. The time differentiation is represented in the conventional manner by use of the frequency multiplier, $u_{0} / \ell_{0}$.

With these estimates of the terms in Eq 15, Eq 18 can be written

$$
\mathrm{P}_{\mathrm{m}} \sim \frac{1}{4 \pi}\left(\frac{\beta \mathrm{K}}{1-\beta \mathrm{K}_{\mathrm{v}}}\right)^{3}\left[\eta\left(\frac{\gamma}{\gamma-1}\right)^{3}-1\right]^{3}\left[\rho_{\mathrm{O}} \sigma \mathrm{U}^{3} \mathrm{~L}^{2}\right][\sigma \mathrm{M}]^{5}\left(\frac{\mathrm{L}}{l_{\mathrm{o}}}\right)
$$


Except for the term

$$
\alpha^{2}=\left(\frac{\beta \mathrm{v}_{\mathrm{v}}}{1-\beta \mathrm{K}_{\mathrm{v}}}\right)^{2}\left[\eta\left(\frac{\gamma}{\gamma-1}\right)^{2}-1\right]^{3}
$$

this is just the power radiated by a typical turbulence quadupole of strength $T_{i j} \sim \rho_{0} \sigma U^{2}$. Hence, the presence of the condensable fog increases the acoustic power output by a factor of $\alpha^{2}$.

Note that the mass loading of droplets does not enter into Eq 17 for the acoustic power. This is an interesting but not wholly unexpected result. Because of the assumption of equilibrium between the droplets and gaseous phase, as long as there is sufficient liquid phase present to maintain the local vapor pressure at its equilibrium value, the actual amount of liquid does not matter. Of course, the assumption regarding droplet size and spacing place other requirements on the nature of the droplet cloud. However, in the absence of finite-rate processes, droplet size and concentration parameters do not appear in the monopole term.

For water vapor in air at $70^{\circ} \mathrm{F}, \mathrm{K}_{\mathrm{v}}=0.02$ and $\alpha^{2} \cong 1.4$. Hence, at ambient conditions, the increase in acoustic power due to the monopole radiation is very slight. However, at $150^{\circ} \mathrm{F}, \mathrm{K}_{\mathrm{v}}=0.25$ and $\alpha^{2} \cong 270$, so that the monopole radiation is much greater. Thus, at elevated temperatures, significant increases in radiation efficiency may occur. Because of the exponential increase of $\mathrm{K}_{\mathrm{v}}$ with temperature, a modest increase in ambient temperature of the jet can cause large increases in acoustic monopole radiation from evaporating droplets.

It should be kept in mind that the above calculations are valid only when the liquid droplets and the gas phase are in equilibrium. Acoustic damping that takes place when the exchange processes between the two phases occur at finite rates is thus excluded from the calculations. In 
addition, the above result, $\mathrm{Eq} 17$, is not valid as $\mathrm{K}_{\mathrm{v}}$, and hence $\beta \mathrm{K}_{\mathrm{v}}$ approaches unity. From Eq 13 this implies that $\frac{\mathrm{DK}_{\mathrm{V}}}{\mathrm{Dt}}=0$ and that $Q$ is, however, finite and not equal to zero. Consideration of the case in which the gas phase consists of pure vapor $\left(\mathrm{K}_{\mathrm{v}}=1\right)$ requires the use of the energy equations for the two phases to determine $Q$. The question of acoustic damping, the dipole and quadrupole terms in $\mathrm{Eq} 3$, and the case for $\mathrm{K}_{\mathrm{v}}=1$ are subjects for further investigation. The above calculation, with the results given by Eq 17 is a first step toward gaining an understanding of sound generation by the turbulent flow of a condensing vapor. 


\section{CONCLUDING REMARKS}

Perhaps the most interesting result obtained to date is that the frequency of the peak observed in the core is a function of velocity but is independent of jet diameter, at least in laboratory-scale jets. This implies that the peak frequency cannot be nondimensionalized as a Strouhal number using the outlet diameter as the characteristic length. The fact that the peak frequency is proportional to the velocity indicates a constant (within experimental error) characteristic length for subsonic jet flow. One such length in the flow is the distance between the intermittent bursts that are observed at the boundary between the core and the turbulent annulus surrounding it. At low Reynolds numbers, this structure takes the shape of doughnutlike vortex rings propagating away from the jet lip, as shown by the excellent flow visualization of Crow and Champagne. ${ }^{19}$ At higher Reynolds numbers, the details of the vortex structure probably are lost, being replaced by a jetlike structure similar to that observed in the outer region of a turbulent boundary layer. ${ }^{24}$ Experiments are continuing at SRI to determine the characteristic length associated with these phenomena.

More consideration must be given to developing a meaningful definition of the convection velocity of turbulent eddies in the shear layer and to its relation to the convection velocity of sound observed in the near field. Future work will also emphasize the feedback between the waves in the core and the jetlike disturbances emanating from the mixing region. Experiments during the next year will be concerned with the effects of swirl on the observed phenomena. Also included will be a continuing study of the effects of a fog of condensible droplets on the noise output. 


\section{RRECEDING BAGE BLANK NOT FLLMED}

\section{REFERENCES}

1. Lighthill, M. J., "Jet Noise," AIAA J., 1, 1507-1517 (1963)

2. Lighthill, M. J., "On Sound Generated Aerodynamically. I. General Theory," Proc. Roy. Soc. (London), A211, 564-587 (1952)

3. Lighthil1, M. J., "On Sound Generated Aerodynamically. II. Turbulence as a Source of Sound," Proc. Roy. Soc. (London), A222, I-32 (1954)

4. Lighthill, M. J., "Sound Generated Aerodynamically," Bakerian Lecture, 1961, Proc. Roy. Soc. (London), A267, 147-182 (1962)

5. Powel1, A., "Concerning the Noise of Turbulent Jets," J. Acoust. Soc. Am., 32 (12), 1609 (1960)

6. Powell, A., "Theory of Vortex Sound," J. Acoust. Soc. Am., $\underline{36}$ (1), 177-195 (1964)

7. Ffowcs Williams, J. E., "On the Development of Mach Waves Radiated by Small Disturbances," J. Fluid Mech., 22, 49-55 (1965)

8. Pfowcs Williams, J. E., "Sound Radiation from Turbulent Boundary Layers Formed on Compliant Surfaces," J. Fluid Mech., 22, 347-358 (1965)

9. Harris, C. M., Handbook of Noise Control, McGraw-Hill, New York, 1957

10. Fitzpatrick, H. M., and R. Lee, "Measurements of Noise Radiated by Subsonic Air Jets," David Taylor Model Basin Report 835, 1952

11. Lassiter, L. W., "Turbulence in Small Air Jets at Exit Velocities up to 705 Feet per Second," J. Appl. Mech., 24, 349-354 (1957)

12. Lee, R., "Free-Field Measurements of Sound Radiated by Subsonic Air Jets," David Taylor Model Basin Report 868, 1953

13. Lassiter, L. W., and H. H. Hubbard, "Experimental Studies of Noise from Subsonic Jets in Still Air," NACA TN 2757, 1952 
14. Laurence, J. C., "Intensity, Scale, and Spectra of Turbulence in the Mixing Region of a Free Subsonic Jet," NACA Report 1292, 1956

15. Laurence, J.C., and J.M. Beninghoff, "Turbulence Measurements in Multiple Interfering Air Jets," NACA TN 4029, 1957

16. Davies, P.O.A.L., M. J. Fisher, and M. J. Barratt, "The Characteristics of the Turbulence in the Mixing Region of a Round Jet," J. Fluid Mech., 22, 337-367 (1963)

17. Davies, P.O.A.L., N.W.M. Ko, and B. Bose, "The Local Pressure Field of Turbulent Jets," ARC-CP 989 (1968)

18. Bradshaw, P., D. H. Ferriss, and R. F. Johnson, "Turbulence in the Noise-producing Region of a Circular Jet," J. Fluid Mech., 19, 591624 (1964)

19. Crow, C. S., and F. H. Champagne, "Orderly Structure in Jet Turbulence," Boeing Sci. Res. Lab., Doc. D1-82-0991 (1970)

20. Wooten, D. C., C. E. Wooldridge, A. J. Amaro, and G. R. Plapp, "A Study of the Structure of Jet Turbulence Producing Jet Noise," Annual Report, NASA Contract NASW-1938, Stanford Research Institute, Menlo Park, California, July 1970

21. Nagamatsu, H. T., R. E. Sheer, and G. Horvay, "Supersonic Jet Noise, Theory and Experiments," Proc. of the Conf. on Basic Aerodynamic Noise Research, NASA SP-207, July 1969

22. Wills, J.A.B., "Measurements of the Wave-Number/Phase Velocity Spectrum of Wall Pressure Beneath a Turbulent Boundary Layer," J. Fluid Mech., 45, 65-90 (1970)

23. Fisher, M. J., and P.O.A.L. Davies, "Correlation Measurements in a Nonfrozen Pattern of Turbulence," J. Fluid Mech., 18, 97-116 (1963?)

24. Willmarth, W. W., and C. E. Wooldridge, "Measurements of the Fluctuating Pressure at the Wall Beneath a Thick Turbulent Boundary Layer," J. of Fluid Mech., 14, 187-210 (1962)

25. Crighton, D. G., and J. E. Ffowcs Williams, "Sound Generation by Turbulent Two-Phase Flow," J. Fluid Mech., 36, 585-603 (1969) 
26. Marble, F. E., "Some Gas Dynamic Problems in the Flow of Condensing Vapor," Astronautica Acta, 14, 585, (1969)

27. Marble, F. E., and D. C. Wooten, "Sound Attenuation in a Condensing Vapor," Phys. Fluids, 13, 2657-2664 (1970) 